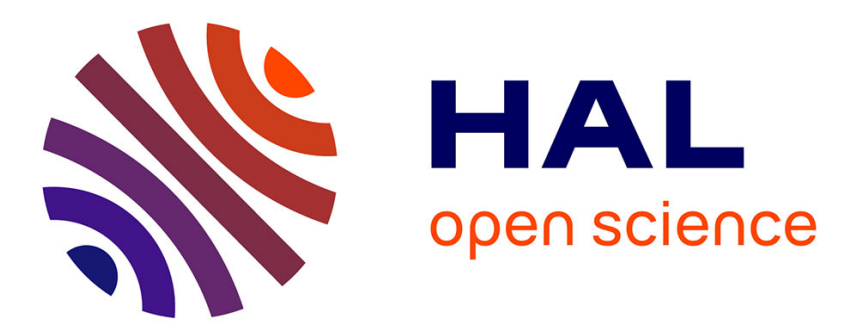

\title{
Comparisons of experimental measurements and large eddy simulations for a helium release in a two vents enclosure.
}

Gilles Bernard-Michel, Elie Saikali, Anne Sergent, Christian Tenaud

\section{To cite this version:}

Gilles Bernard-Michel, Elie Saikali, Anne Sergent, Christian Tenaud. Comparisons of experimental measurements and large eddy simulations for a helium release in a two vents enclosure.. International Journal of Hydrogen Energy, 2019, 44 (17), pp.8935-8953. 10.1016/j.ijhydene.2018.07.120 . hal02086322

\section{HAL Id: hal-02086322 \\ https://hal.science/hal-02086322}

Submitted on 1 Apr 2019

HAL is a multi-disciplinary open access archive for the deposit and dissemination of scientific research documents, whether they are published or not. The documents may come from teaching and research institutions in France or abroad, or from public or private research centers.
L'archive ouverte pluridisciplinaire $\mathbf{H A L}$, est destinée au dépôt et à la diffusion de documents scientifiques de niveau recherche, publiés ou non, émanant des établissements d'enseignement et de recherche français ou étrangers, des laboratoires publics ou privés. 


\title{
COMPARISONS OF EXPERIMENTAL MEASUREMENTS AND LARGE EDDY SIMULATIONS FOR A HELIUM RELEASE IN A TWO VENTS ENCLOSURE
}

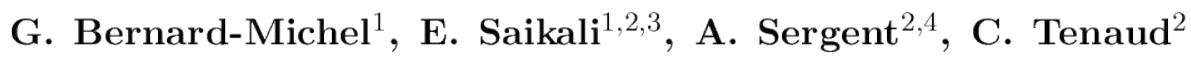 \\ 1 D.E.N, D.A.N.S, S.T.M.F, \\ L.E.I.F.T, Bât. \& 60 , \\ CEA Saclay, Gif-Sur-Yvette, France \\ e-mail : gilles.bernard-michel@cea.fr \\ 2 LIMSI, CNRS, Universit Paris-Saclay, \\ Bât. 508, F-91405 Orsay, France \\ e-mail : elie.saikali@limsi.fr, \\ anne.sergent@limsi.fr, christian.tenaud@limsi.fr \\ 3 Sorbonne Université, Collège Doctoral, \\ ED391 SMAER, F-75006, Paris, France \\ 4 Sorbonne Université, UFR 919 Ingénierie, \\ F-75005, Paris, France
}

\begin{abstract}
This work takes place in the context of potential hazards in the use of hydrogen in fuel cells. The present article describes comparisons between PIV measurements performed on a two vented cavity with an helium injection and Large Eddy Simulation of the same configuration. A two vented cavity is chosen because a quasi state state is reached rapidly and it facilitates both CFD calculations by reducing the CPU costs and also enable statistical treatment of the data, the temporal averaging being possible at steady state. At the same time, this configuration is close to fuel cell designs, except for the set-up reduced size. We also describe the experimental set-up and the care which has to be taken to produce Particle Image Velocimetry velocity fields. The final goal of the paper is to validate L.E.S approach as a good replacement to experiments, since access to both velocity and concentration fields is required to improve existing simplified models. Indeed, most of the 2 vents models rely on simplified assumptions such as a constant entrainment coefficient, a bi-layer formation which is not always the case in real situations.
\end{abstract}

\section{CONTEXT}

Accidental releases of hydrogen in a fuel cell lead to the formation of a dispersed cloud of hydrogen and air which is potentially explosive when hydrogen volumic concentration is above $4 \%$. Many experimental studies are based on helium release and dispersion as a safe replacement for hydrogen as it proved to show the same behavior [1]. Accurate experimental measurements of helium concentration dispersion have been performed by several authors $[2-7]$ in large scale enclosures equipped with none, one or two ventilation openings. Those experimental results have been compared to simplified analytical models $[6,8-10]$ with satisfactory agreements. Nevertheless, discrepancies were found in some cases $[3,5]$ and could not be explained without an access to the velocity field. Finally, in order to address those questions, two approaches can be considered: either to use CFD 
calculations or to measure the velocity fields in the experiments (Particle Image Velocimetry for example). Different benchmarks have been published on closed, one vented and two vented cavities [11-18]. They showed satisfactory agreement between CFD and experimental concentration measurements for jet releases of helium. On the other hand, for plume releases, R.A.N.S models for turbulence proved to be significantly more diffusive than measurements [13, 15]. Only "coarse" Large Eddy Simulation with Smagorinsky models for turbulence capture correctly the maximum concentration [18] but with still a strong diffusion for the minimum concentrations (being higher than expected).

On the experimental side, PIV measurements can mostly been achieved on small set-ups, the maximum reasonable size of a velocity field being around $40 \mathrm{~cm}$ with a $8 \mathrm{Mpx}$ PIV camera. Very few measurement exist on a free jet at a meter scale, and to our knowledge none on a set-up as large as a fuel cell. As a result, we decided to develop two types of research jointly: to characterize the flow in vented cavities through highly resolved L.E.S numerical simulations $[19,20]$ and to perform PIV measurements of the flow in a small enough experimental set-up. This volume limitation is actually of the same order for the CFD approach due to the need to represent spatial scales close to the Kolmogorov length.

In this paper, we present an experimental set-up designed to perform injection and dispersion of helium in a 2 vented box with Particle image velocimetry measurements. Comparisons between experimental measurements and numerical results obtained for the same configuration [21] are presented. This article largely validate the L.E.S numerical approach although some difficulties are shown to remain.

The first section of this paper presents briefly the context of this work. In the second section the experimental setup is described. In the third part, statistical operators and statistical independence of the data is discussed. At last, in the fourth part, the numerical approach is briefly and comparisons between experimental and numerical results are discussed.

\section{EXPERIMENTAL SETUP}

\section{A. Choice of the experimental set-up}

The experiment is carried out on a parallelepiped cavity. In order to reach rapidly a quasi steady state, two vents are considered inside the cavity and located respectively at the bottom and at the top of the same solid wall boundary, figure 1 .

The height of the set up has been set to $15 \mathrm{~cm}$ in order to have a high spatial accuracy of the PIV measurements. Indeed with a $8 \mathrm{Mpx}$ camera and a window size of $16 \mathrm{px}$, the physical size of the window is around $0.6 \mathrm{~mm}$ which is about 3 times the Kolmogorov scale. With a window shift of $8 \mathrm{px}$, we have access to information very close to the Kolmogorov scale. The width of the set-up is chosen around $5 \mathrm{~cm}$, which is slightly more than the expected turbulent spreading of the plume in order to limit jet/wall interaction. After machining of the box panels in Plexiglas, the internal dimensions of the cavity are $W \times L \times H=4.9 \times 5 \times 14.9 \times 10^{-6} \mathrm{~m}^{3}$. 

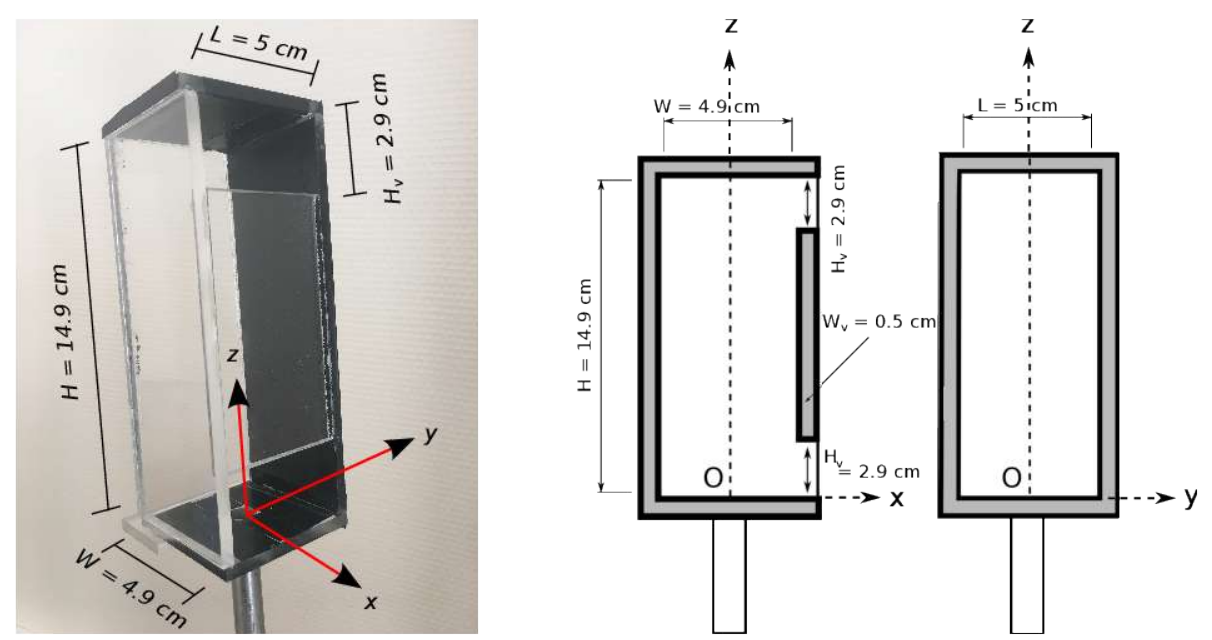

FIG. 1: Experimental set-up

The injection diameter of $10 \mathrm{~mm}$ and the flow rate of $5 \mathrm{NL} / \mathrm{min}$ were chosen to ensure a laminar to turbulent transition in the mid height of the enclosure predicted to occur at $H / d>5$ [22], as well as a jet to plume transition. The injection pipe length is over 30 diameters long and a laminar Poiseuille flow is insured upwind at the inlet centered at the base of the box.

At last, both vents size was chosen equal and to be smaller than the expected so called Linden's top homogeneous layer [10], with a surface area $5 \times 2.9 \times 10^{-4} \mathrm{~m}^{2}$. We will see in the results section that this Linden bi-layer structure doesn't occur in this configuration (on going work shows that a higher injection flow rate is required).

Plexi-glass of thickness $5 \times 10^{-3} \mathrm{~m}$ is used for the solid wall boundaries.

\section{B. $3 / 8 \mathbf{m}^{3}$ experimental configuration}

Environment : This experimental set-up is located inside a $30 \mathrm{~m}^{3}$ garage, Fig. 2, fully isolated with styridur panels. Temperature variations monitored with a $\mathrm{Pt} 100$ sensors are below $0.1^{\circ} \mathrm{C}$ over an hour. Daily temperature variation is below $0.5^{\circ} \mathrm{C}$ and temperature is monitored during experiments. The garage itself is part of a warehouse with small daily temperature fluctuations $\left(1{ }^{\circ} \mathrm{C}\right.$ on average). The garage is also fully sealed, except with the presence of two circular vents of $30 \mathrm{~cm}$ diameter in order to evacuate potential accumulation of helium. Residual velocities in the garage are not measurable in comparison with the velocities at the experimental set-up vents (which are the entry/exit interfaces between the box and the room), that is lower than a few $\mathrm{cm} / \mathrm{s}$. We notice that the laser power supplies have been placed outside of the garage to reduce the potential natural convection that could have been induced if located inside the garage.

Experimental build-up : The cavity and the whole instrumentation, that is camera and laser are tighten together with Bosch aluminium profiles, figure 3. This ensures the required perpendicularity of the cavity main axis, the camera and the laser light sheet. The verticality of the system can be also easily been ensured for all the equipments. 


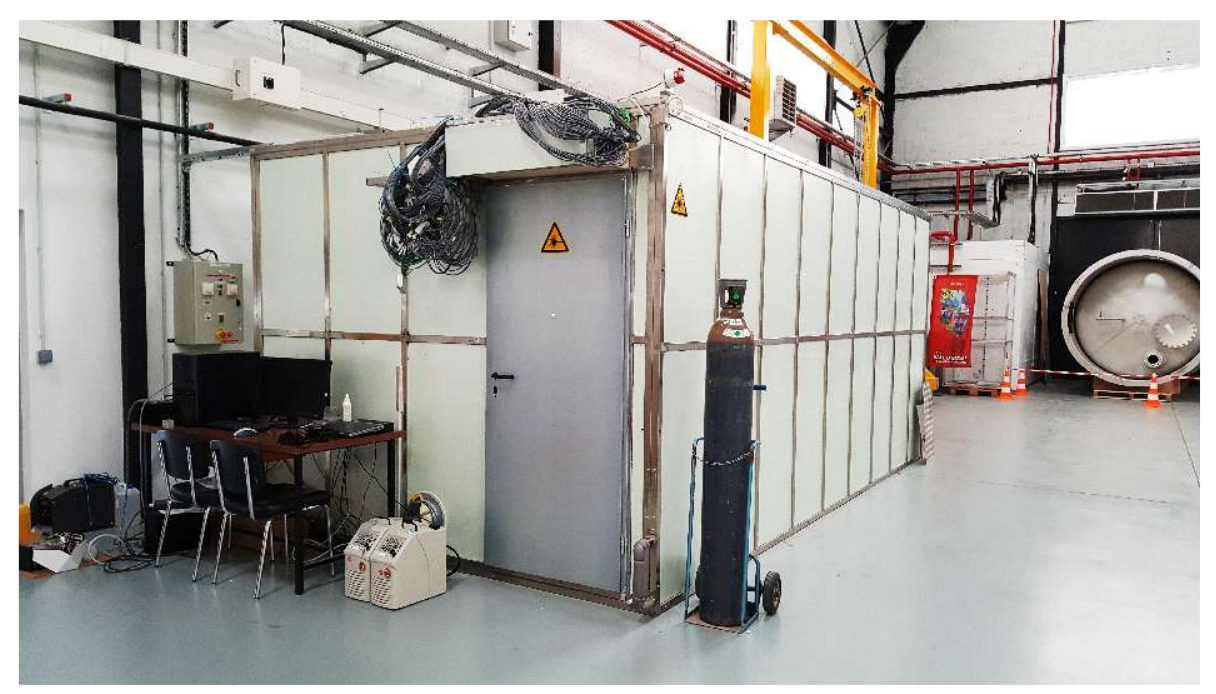

FIG. 2: Garage where is located the experimental set-up.

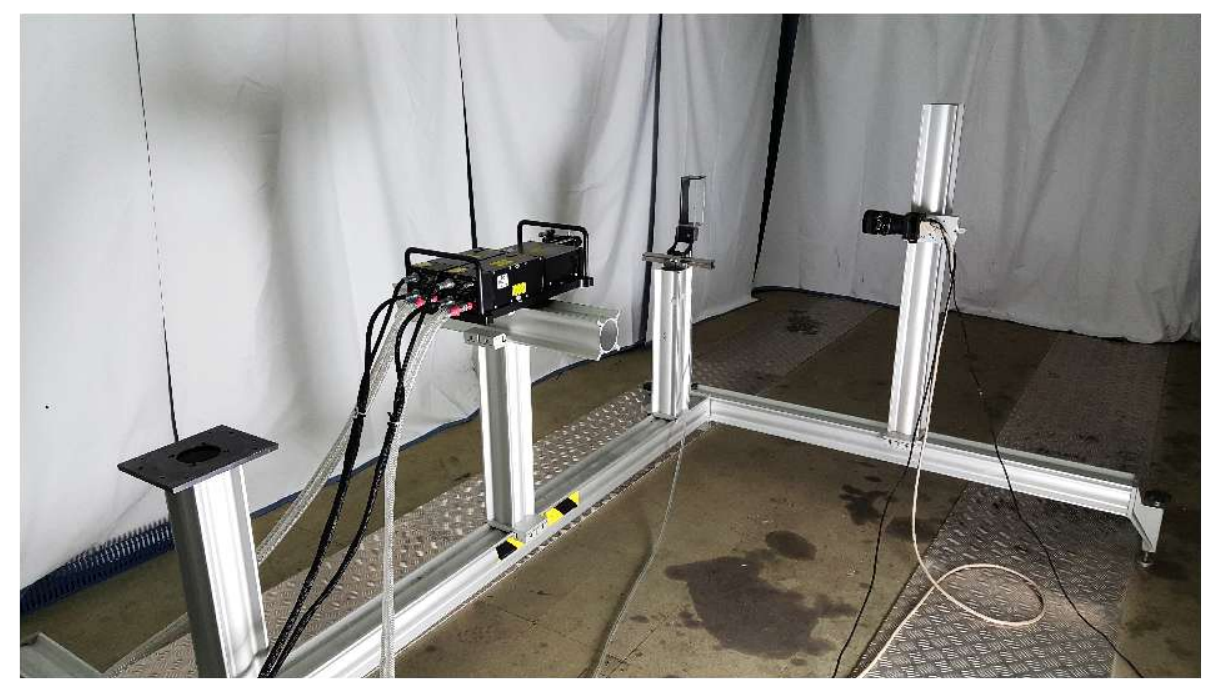

FIG. 3: Experimental set-up with Bosch aluminium profiles for assembly. Quantum pulsed Yag laser is presented.

Injection : we inject pure helium through a cylindrical pipe with a unique constant mass flow-rate $Q=5 \mathrm{Nl} / \mathrm{min}$ into a two vented parallelepiped enclosure filled initially with air at rest. NormoLiters per minute is a mass flow rate in that it is the volumetric flow rate that would occur at $0^{\circ} \mathrm{C}$ injection's temperature. At the $25^{\circ} \mathrm{C}$ injection temperature of our experiments, the volumetric flow rate of helium is therefore $Q=5 \times(273+25) / 273=5.45 \mathrm{l} / \mathrm{min}$. That mass flow rate is controlled with a Brooks (1-20 NL/min range) gaz flow controller. That mass control-er had been calibrated within the last 6 months and ensures an accuracy better than $1 \%$ error at the chosen flow rate.

Physical properties : all the physical properties of the light helium gas are referred to with the in subscript (meaning "injected"), while the subscript am is used to denote the ambient air. Both injection temperature and garage temperature have been checked to be at $25^{\circ} \mathrm{C}$ within the error limit of the sensor (around $1^{\circ} \mathrm{C}$ for portable TC used at beginning of experiments, fixed Pt100 were used for monitoring during experiments and set-up qualification). 


\begin{tabular}{cccc}
\hline \hline Fluid & $\begin{array}{c}\text { Density } \\
{\left[\mathrm{kg} \cdot \mathrm{m}^{-3}\right]}\end{array}$ & $\begin{array}{c}\text { Dynamic viscosity } \\
{\left[\times 10^{-5} \mathrm{~kg} \cdot \mathrm{m}^{-1} \cdot \mathrm{s}^{-1}\right]}\end{array}$ & $\begin{array}{c}\text { Molar mass } \\
{\left[\times 10^{-2} \mathrm{~kg} \cdot \mathrm{mol}^{-1}\right]}\end{array}$ \\
\hline Injected & $\rho_{\text {in }}=0.16148$ & $\mu_{\text {in }}=1.918$ & $M_{\text {in }}=0.4003$ \\
Ambient & $\rho_{\text {am }}=1.16864$ & $\mu_{a m}=1.792$ & $M_{a m}=2.897$ \\
\hline
\end{tabular}

TABLE I: Physical properties of the working fluids

Optical devices : the laser beam is produced either by a double cavity 200mJ Yag laser (Quantum), or a continuous 5 Watts laser. The laser beam is then changed into a plan with a semi-cylindrical lens and thickness of the plan is adjusted with a divergent and a convergent lenses. Those lenses are fixed on the quantum laser exit, or on a specific bench screwed to the Bosch profiles. Positioning of the different systems is done with micro-metric screws. With the 2 cavities pulsed yag laser, a PIV 8Mpx camera is used (CMOS) and with the continuous laser, a $1 \mathrm{Mpx} 2 \mathrm{kHz}$ camera is used (cinevision). Lenses used on the camera are full frame designed Carl Zeiss $35 \mathrm{~mm}, 50 \mathrm{~mm}$ and $85 \mathrm{~mm}$ with wide opening (better than 2). Those expensive lenses have very reduced distortion (less than $0.7 \%$ geometrical deformation at the edges). In practise, the geometrical distortion is even lower due to the reduced size of the sensor (half the full frame size). PIV measurements won't therefore be affected by the geometrical distortion. At last the semi-cylindrical lens can rotate in order to adjust the verticality of the laser plan.

Seeding : particles are needed in order to visualise the flow and carry on with PIV measurements. The seeding is realized with a theater smoke generator. The smoke is made of around $1 \mu \mathrm{m}$ droplets, which last around 15 minutes before evaporation. Their size is small enough to ensure no drift in our experimental condition and their motion will represent accurately the gas flow in the room and in the cavity. Indeed, rapidly the fog is sucked into the box through the bottom vent and rejected through the top vent. Droplets volume concentration is such that roughly 10 drops can be seen on a PIV sub-window of $600 \mu \mathrm{m}$, that requires a spacing of approximately $200 \mu \mathrm{m}$ between droplets, that is 200 diameters. The required volume fraction is therefore roughly $1 / 200^{3}$ which is close to $10^{-5} \%$. We can assert that during experiments, image control shows than we never exceed a concentration of $10^{-4 \%}$. We underline this to assert that fog has no significant influence on the gas volumic mass, it would modify it by less tan $0.1 \%$. Fog influence is therefore negligible compared to helium influence on the mixture density.

\section{PIV specifications}

A laser sheet of less than $1 \mathrm{~mm}$ thickness is enlightening a vertical plan, Figure 4 . The fog droplets are enlightened by the laser. For the Yag pulsed laser, the pulse lasts $6 \mathrm{~ns}$ and freezes the droplets at their positions. For the continuous laser, the fast camera shutter can be adjusted from 2 to $10 \mathrm{khz}$ at the cost of a loss of light on the images. In practise we limit ourselves to a $3 \mathrm{khz}$ shutter speed. Particle movements are not frozen, and droplets are seen as short spheres or even lines instead of dots. This doesn't affect too much in practise the quality of the results although it may theoretically reduce the measurements accuracy. 


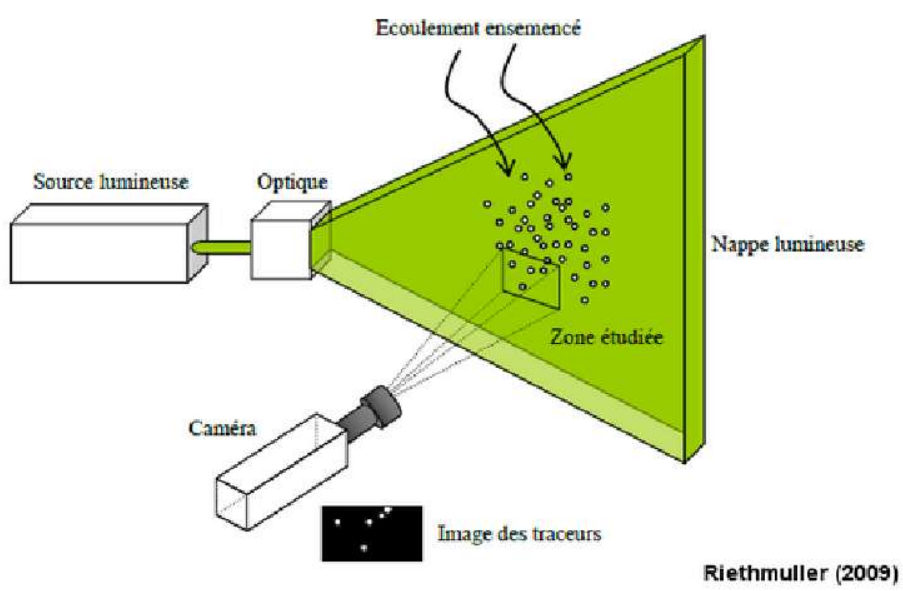

FIG. 4: Illustration of the laser plan creation and camera measurements.

As said, the pulsed laser $(10 \mathrm{~Hz})$ in used in association with a $8 \mathrm{Mpx}$ PIV camera, whereas the continuous laser is used with a $1 \mathrm{Mpx}$ fast camera $(2 \mathrm{Khz})$. With the fast camera we perform measurement at the injection and also near the bottom vent. With the PIV camera, we cover the full enclosure but with slow frequency acquisitions of $1 \mathrm{~Hz}$ (to perform time averaged statistics). But every acquisition is made of two pictures separated by a small time interval ( 250 up to $750 \mu \mathrm{s}$ ). The PIV process consists in splitting each camera pictures in sub-windows. We use 16x16 pixels windows. Those windows might overlap and we choose a 8 pixels overlapping. A pair of images separated by a time interval is selected. Cross correlations of the light signal are done on each pairs of sub-windows, Fig. 5. The cross correlation signal contains a principal peak (maximum value) and secondary peaks. The location of the principal peak is the most probable displacement of the particles in the sub-window. The velocity is obtained by dividing with the time interval.
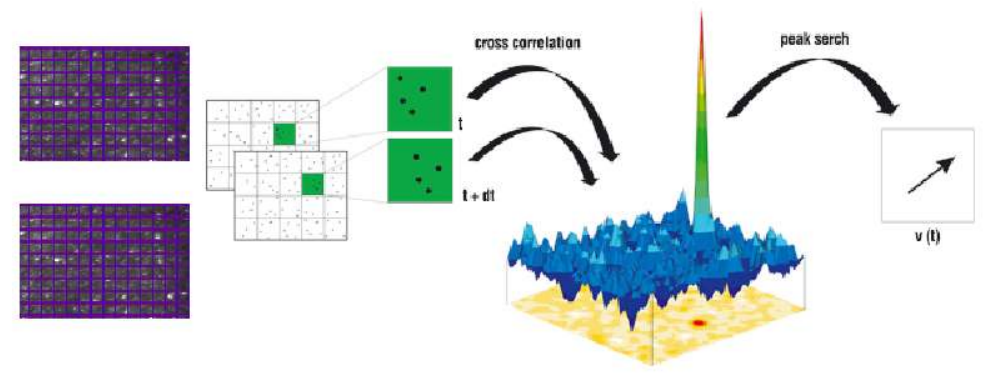

FIG. 5: Illustration of cross correlation calculations and peaks of most probable displacement.

It is important to note that accuracy of the measurement depends on the displacement of the particles on the image. Theories indicate that the position of the peak might be determined with sub-pixel accuracy (around 0.1 to 0.2 pixel). Therefore a 10 pixels displacement of the correlation peak will lead to a 1 to $2 \%$ error. A smaller displacement will evidently lead to higher errors: the smaller displacement, the higher relative error. Nevertheless the absolute error remain the same. That is why we want to select time intervals between pictures that maximise the displacement of 
particles on the sub-windows. On the other hand, a too large displacement leads to the risk of particles leaving the window before the second picture is taken. The correlation peak will correlate first image particles with particles on second image which have entered the window (other particles in fact). The correlation peak position will be a wrong indication of the particles displacement. Therefore two opposite notions coexist: accuracy of the displacement calculation and probability to measure the right displacement.

At last, the picture's quality is of importance as well. If too many particles exist on the picture, they might hide each other and correlation between two successive pictures might be incorrect. If too few exist, errors connected to leaving or entering of particles between pairs of image might also lead to errors. On average we try to seed the ambient gas in order to have around 10 particles on the subwindows. Practically, we calculate velocity fields using the free software GPIV delivered on Ubuntu Linux distributions. We use 16 x 16 px sub-frames with a 8 px shift and a predictor/corrector algorithm with distorted images capability. Basically, an estimate of the velocity is made with the procedure described above, then sub-framed are moved and distorted according to that first prediction. A new velocity field is calculated and an iterative process is engaged until converged to a certain accuracy.

\section{Measurement accuracy}

Image quality : In order to improve the final image quality, we operate different treatments. The first treatment is to calculate an image where at each pixel, we calculate the minimum pixel value of all the images. we then subtract this image to all the original images. This helps to remove all the background light and light reflexions on the picture. We also replace all the walls (where there is obviously no flow) by the a fixed random image of particles. This helps to post-treat the images with no artifacts in the wall regions, where velocities will be calculated as zero thanks to the fixed particles artificially located in the walls. No other treatment (luminosity, contrast etc...) is done since it proved to be useless.

Time steps adjustment : The second important choice to make is the time step between to images forming a pair. For the fast camera, the frequency of acquisition is $2 \mathrm{kHz}$. We can pair successive images or 1 every 3 or more pictures. In practise, we pair every successive images when we are interested at the velocities in the jet, the time step is therefore of $500 \mu \mathrm{s}$. For the flow fields around the bottom vent, we select a time step of $1 \mathrm{~ms}$ by dropping 1 every 2 pictures. We have analysed more thoroughly the selection of time steps with the yag cavity laser since time interval between cavities can be chosen as wished. We calculated that a $250 \mu$ s time step is ideal for the jet region with largest velocities (around 1 up to $2 \mathrm{~m} / \mathrm{s}$ ), whereas a time step of $750 \mu \mathrm{s}$ is best suited for the outer jet region where velocities range between 0.1 up to $0.5 \mathrm{~m} / \mathrm{s}$. We therefore have to calculate twice the full picture fields and collect the velocity fields in the regions depending on the time step used.

Signal to noise ratio : that parameter indicates the ratio between the maximum value of the first correlation peak and the second peak maximum value. GPIV software opts the inverse ratio, the smaller the value, the most probability to measure the correct velocity. We plot an example of the signal to noise ratio on a 2D plan, Fig. 6. The GPIV signal to noise ratio is close or higher to one in the regions where velocity is maximum. Is is expected since particles might exit the sub-windows. 
Furthermore it has to be noticed that a small region near the injection hardly contains particles (due to the injection of pure helium and no mixing with the air has happened yet). This might also lead to bad SNR in the vicinity of injection. In practise we consider the region located up to $2 \mathrm{~cm}$ above region as unusable for measurements. We won't compare PIV results with CFD results in that region, although plots will contain that region.
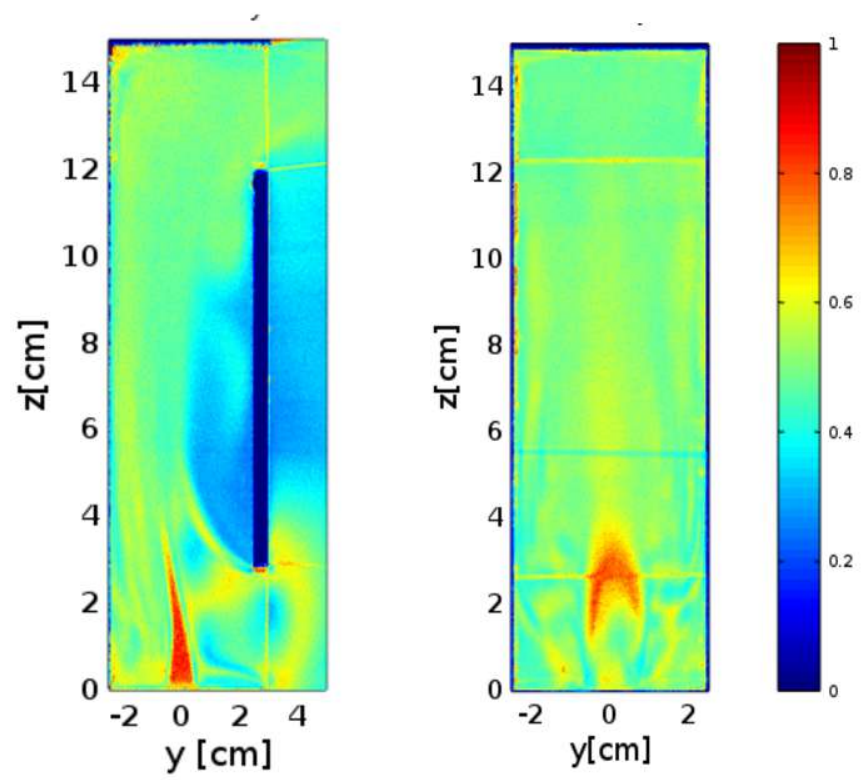

FIG. 6: Example of a signal to noise ratios distributions in $y=0$ and $x=-0.5$ plans.

We assessed that almost $95 \%$ of the data are associated with a GPIV SNR below 1 (that is a standard SNR above 1). We also plot, Fig. 7, the time averaged velocities calculated versus the GPIV SNR. For a given GPIV SNR, we include all the data with a lower GPIV SNR to calculate the average. We observe that for a GPIV SNR below 1, the average is not modified. For GPIV SNR above 1 , the average is modified but only by less than $1 \%$. For data associated with a GPIV SNR higher than 1, velocities may be wrong (by a 100\%) but they only merely contribute to an error on the time averages. This is due to the fact that less than $5 \%$ of the data are concerned. Nevertheless, only data with a GPIV SNRS lower than 1 will be used to calculate the statistical quantities. This is a common procedure in PIV techniques.
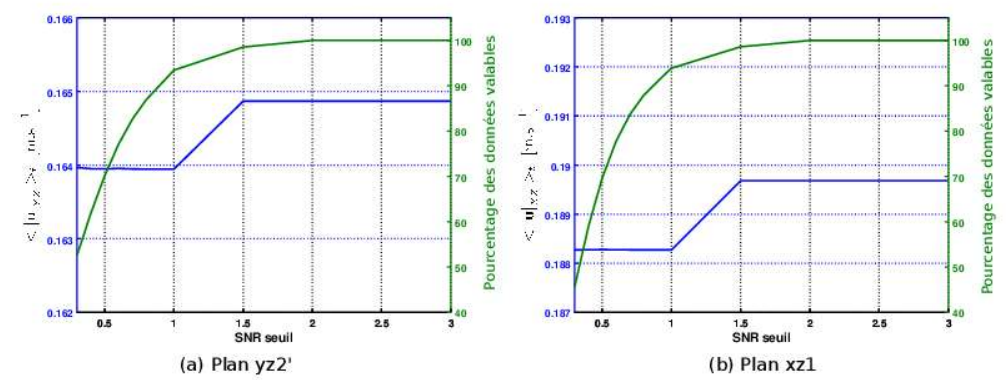

FIG. 7: Test of the influence of GPIV SNRS Thresold on time averaged velocities. 2 laser plans are selected for illustration.

Steadiness : we are interested in quasi steady states. Although it has no connection with PIV accuracy, it is also an important aspect of experiments qualification to ensure that our data are 
indeed produced during a quasi steady state. We will discuss more in depth this aspect in the next section.

Measurement errors : All the preceding qualifications lead to expect an absolute accuracy better than 0.2px for the displacement calculations with a 95\% confidence. Our time steps strategy, based on separating varying regions of the flow based on the velocity intensity, lead to an expected absolute error of $8 \mathrm{~cm} / \mathrm{s}$ in high velocity regions (the maximum velocity being around $2 \mathrm{~m} / \mathrm{s}$ ) and an absolute error lower than $3 \mathrm{~cm} / \mathrm{s}$ in the lowest velocity regions (velocities range between 0 up to $0.6 \mathrm{~m} / \mathrm{s})$.

Time aliasing : we checked that no slow periodicity/aliasing phenomenon interfere when doing acquisition at $1 \mathrm{~Hz}$ with the pulsed laser. We performed up to $7 \mathrm{~Hz}$ acquisitions with the pulsed laser and $2 \mathrm{khz}$ acquisitions on a fast camera which excluded that results at $1 \mathrm{~Hz}$ could be affected by such a problem.

\section{E. Experimental process}

Reproducibility : a strong attention has been put on reproducibility of the results. Each experiment has been done many times with different types of material (cameras, lasers, fog generators), at different places (the garage has been moved from one warehouse to another) and different seasons (slight temperature differences of a few degrees). Results are always reproduced within the expected experimental error.

We illustrate, Fig. 8, velocity profiles in the vertical plan perpendicular to the vent centered on the jet axis $(x o z)$, for different heights. Two repeated experiments are compared, and maximum discrepancies on the averages velocities are always below $3 \mathrm{~cm} / \mathrm{s}$ locally, which is coherent with the expected accuracy of the measurements.

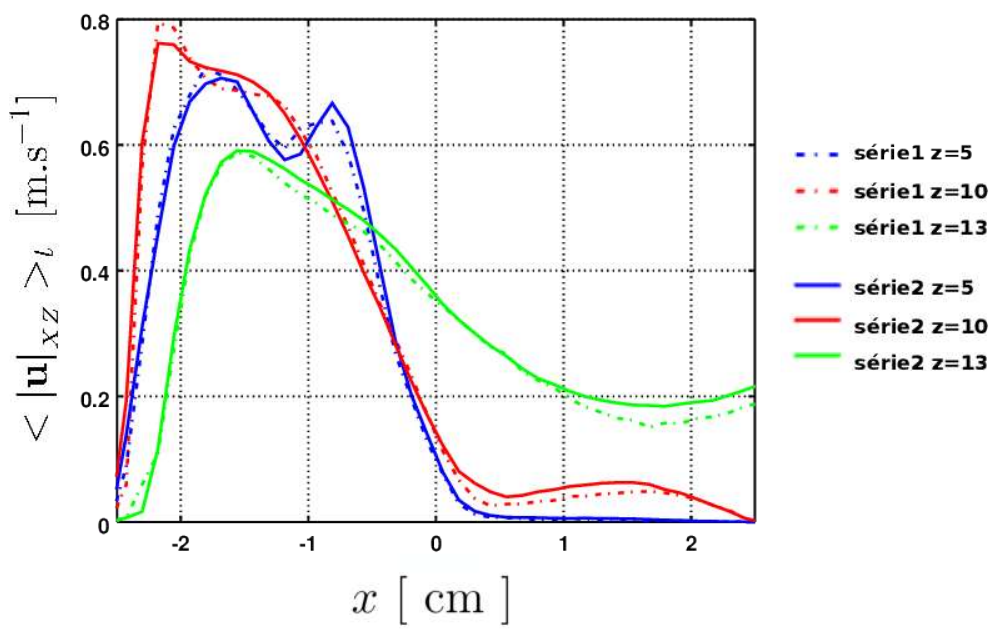

FIG. 8: Reproducibility of the results on 2 tests. Time averaged velocity norm is plotted at 3 heights of the $\mathrm{xOz}$ plan.

In order to achieve this kind of result, we had to take care of the following points: image quality, experimental set-up geometrical alignments, experimental conditions, quasi steadiness. 
Image quality: we have already mentioned that pre process was made on the obtained pictures to remove background noise, and amount of seeding has to be thoroughly controlled. We repeat the following steps:

- We use a smoke generator for a few seconds in the garage.

- After smoke generation, we mix the garage atmosphere with a mechanical ventilation.

This process has probably a minor impact on reproducibility but is applied anyway.

Experimental set-up alignment : we already mentioned that using Bosh profile helped to ensure the correct angles and verticality of the system. This is of great importance, we have notice that a slightly non vertical system (as slight as half a degree) has an impact on the expected plan symmetries of the results. Also, it is very important to position the laser plan where it should. We use micrometer screws and light sensitive paper to accurately position the laser plan. We expect an accuracy better than $1 \mathrm{~mm}$ in the positioning which is almost the thickness of the laser plan.

Experimental conditions : it mainly consists in controlling a steady temperature and the absence of perturbing air movement in the GARAGE. As we said the GARAGE is well insulated and sealed. Residual velocities should dissipate in a few minutes, especially with the absence of heat source and ventilation in the room (power supplies, PCs are located outside).

Steadiness : In order to reach and record data during a steady state :

- Acquisition starts 5 minutes after closing the access door of the garage.

- Steady state will be analysed in post treatment and final considered sequence might start sometimes between 5 up to 10 minutes after smoke injection.

- helium is injected at the same time as the closing of the garage (the steady state is control ed both by helium injection start and the time unwanted air movement in the garage become negligible).

Scaling factor : at last, in order to convert dimensions in pixel into real lengths, we have to evaluate the scale factor which is the real equivalent size of 1 pixel. In order to do that we place a meter in the laser plan (it is very important that this meter is located in the plan where the lens focus is done, since focusing slightly modifies the focal length and therefore the image zooming). This operation must be redone for each experiment, Fig. 9. Geometrical dimension can be estimated to an accuracy better than $0.5 \mathrm{~mm}$ and therefore, the scaling factor error is lower than $1 \%$. The scaling error leads to a multiplicative error on the calculated pixel/s velocities. It is a global error. It is impossible to guess on the PIV pictures where are the boundaries of the box due to multiple reflections, and therefore impossible to derive this scaling factor correctly. We made around $10 \%$ errors when we initially operated without a meter picture for each experiment. 


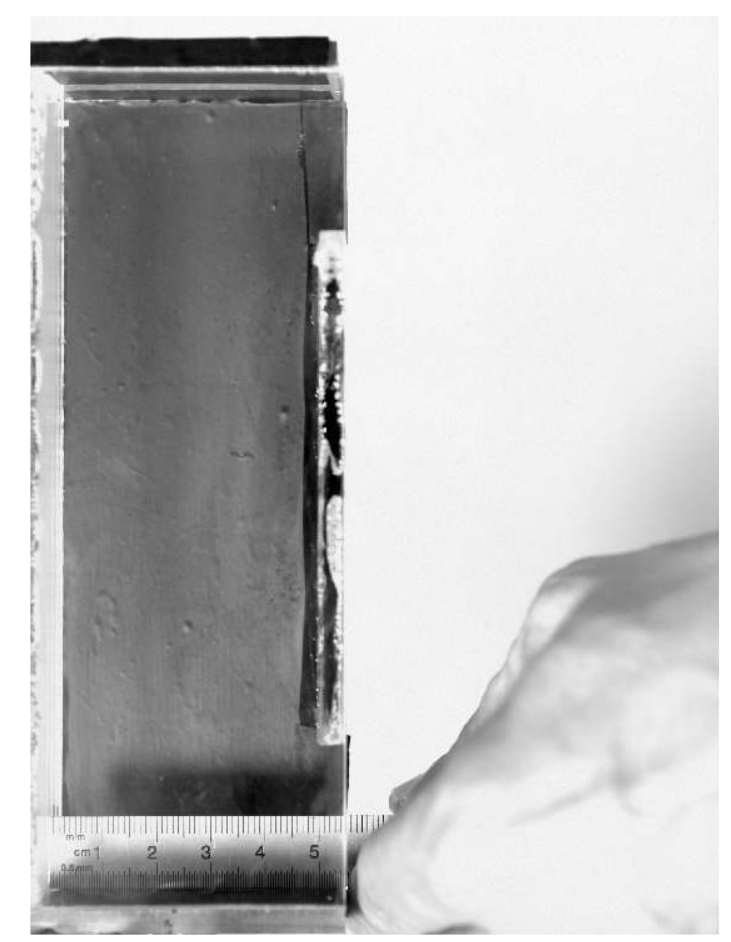

FIG. 9: Meter image to calculate the scaling factor.

\section{F. 2D slices for contour plots}

Results of CFD and experiments are compared on 14 selected vertical two dimensional (2D) slices in the horizontal and span-wise directions, respectively $x$ and $y$. The slices along the horizontal dimension are denoted by $\mathrm{XZ}_{i}$, while $\mathrm{YZ}_{j}$ is used for slices along the span-wise direction. Locations of the slices are materialized on sub-figure (a) of figure 10 from a top view schematic representation, or on sub-figure (b) of figure 10 for a complete three dimensional (3D) observation. For completeness, a detailed description is reported in table II. CFD will provide the 3 components of the velocity in each of the selected plans, whereas PIV measurements only provide $2 \mathrm{D}$ components of the velocity in the plans. The orthogonal component hasn't been measured due to the difficulty to use stereo PIV on such a small cavity.

\begin{tabular}{|c|c|c|}
\hline \multirow{8}{*}{ 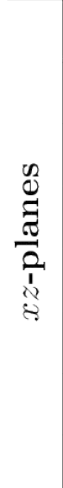 } & Slice & $y$-position $[\mathrm{cm}]$ \\
\hline & $X Z_{1}$ & $y=0$ \\
\hline & $X Z_{2}$ & $y=0.5$ \\
\hline & $X Z_{3}$ & $y=1.5$ \\
\hline & $X Z_{4}$ & $y=2$ \\
\hline & $X Z_{2^{\prime}}$ & $y=-0.5$ \\
\hline & $X Z_{3^{\prime}}$ & $y=-1.5$ \\
\hline & $X Z_{4^{\prime}}$ & $y=-2$ \\
\hline
\end{tabular}

\begin{tabular}{|c|c|c|}
\hline \multirow{8}{*}{ 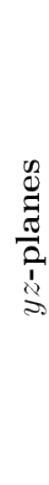 } & Slice & $x$-position $[\mathrm{cm}]$ \\
\hline & $Y Z_{1}$ & $x=0$ \\
\hline & $Y Z_{2}$ & $x=0.5$ \\
\hline & $Y Z_{3}$ & $x=1.5$ \\
\hline & $Y Z_{4}$ & $x=2$ \\
\hline & $Y Z_{2^{\prime}}$ & $x=-0.5$ \\
\hline & $Y Z_{3^{\prime}}$ & $x=-1.5$ \\
\hline & $Y Z_{4^{\prime}}$ & $x=-2$ \\
\hline
\end{tabular}

TABLE II: Position of the PIV studied vertical slices: Left: $x z$-plane, right: $y z$-plane. 


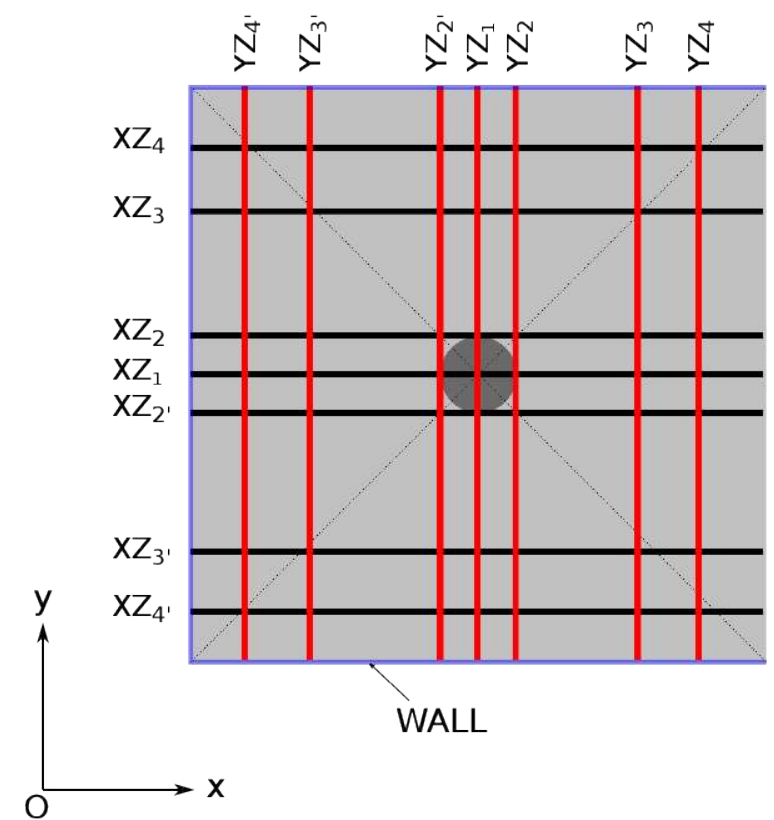

(a)

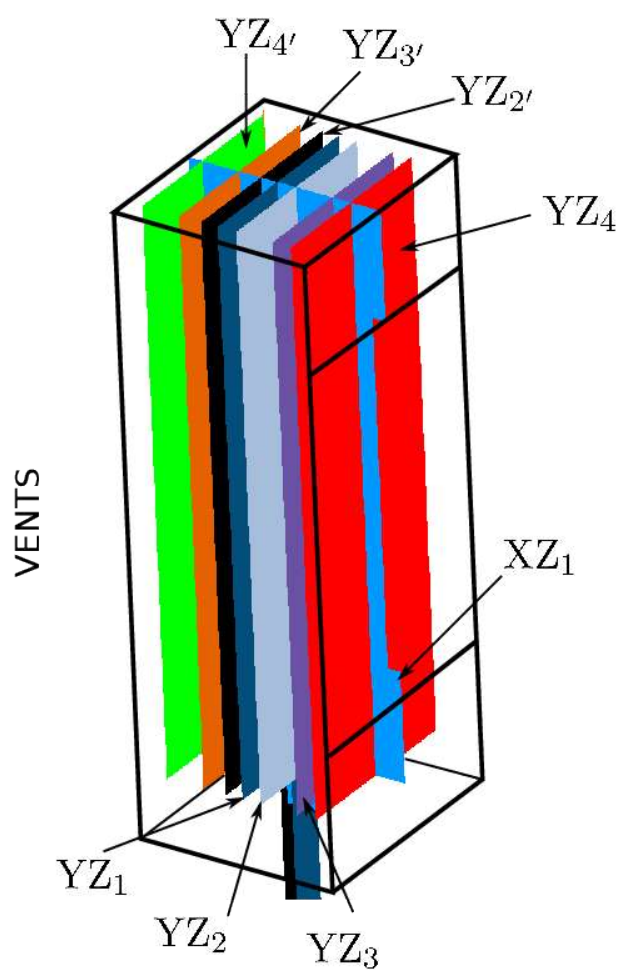

(b)

FIG. 10: Schematic representation of the vertical slices used in the PIV measurements. Left: 2D top-cavity view, right: 3D representation for some selected slices.

Experiments have therefore been performed for each of the selected plans, whereas a single CFD calculation could be used to restrict the results on each of the selected plans.

\section{STATISTICAL POST TREATMENTS}

Results produced both by experimental measurements and with a numerical simulation have to be post treated in a same manner. Since we are only interested in the quasi steady state, we have to ensure first that we are in such a state, and second to calculate time averaged quantities and rms values of the time fluctuations. At last, in order to evaluate the statistical accuracy of the data, we need to characterize the time correlation lengths which will determine the number of uncorrelated data.

\section{A. Quasi steady state}

In order to check that we obtain a quasi steady state, each experiment or CFD calculation has to be reviewed individually. Of course, once we have established a time after which steadiness is observed, it remains more or less the same for all the others (since only the laser map location changes for example). Nevertheless data have always to be reviewed to avoid the presence of 
unpredicted errors. Steadiness is checked by plotting the time evolution of the velocity norm at different locations in the enclosure, Fig. 11.
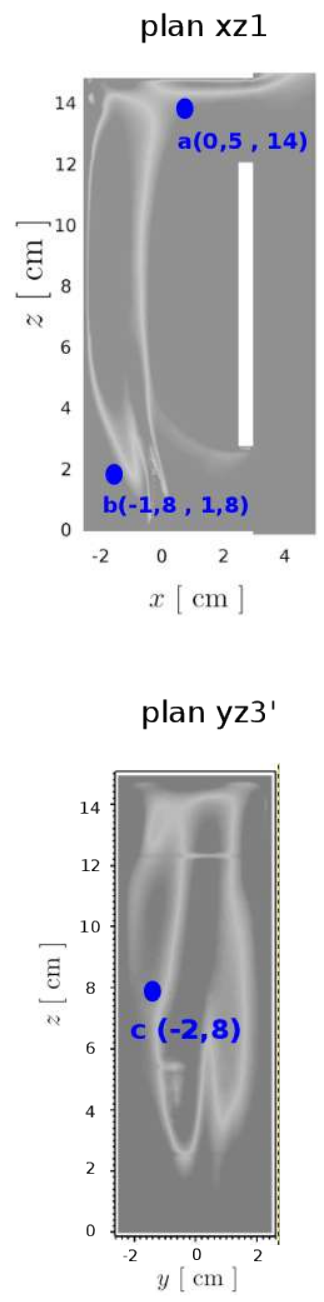

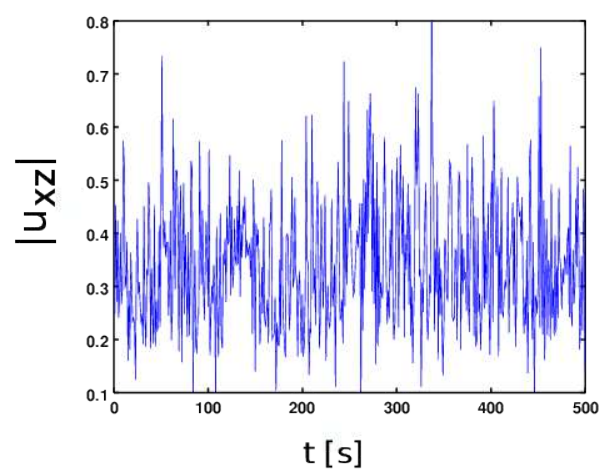

(a)

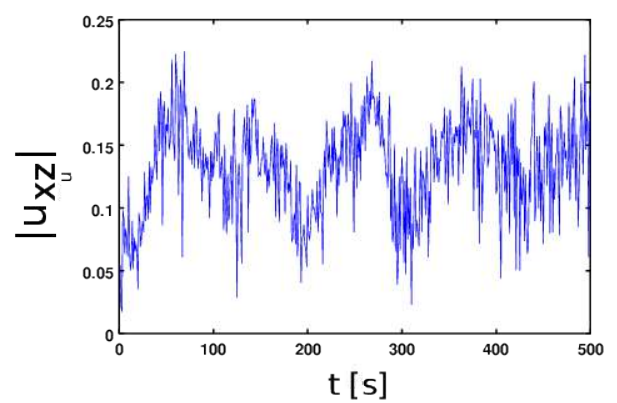

(b)

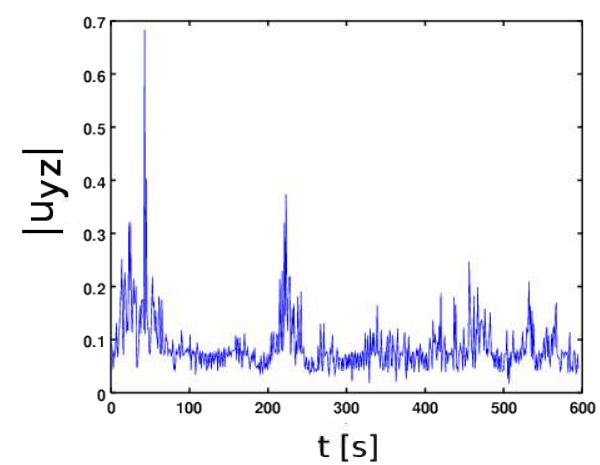

(c)

FIG. 11: Examples of time evolutions of the norm $|\mathbf{u}|_{a}$ at three locations (a) $(\mathrm{x}=0,5 \mathrm{~cm}, \mathrm{z}=14 \mathrm{~cm})$, $\mathrm{xz}_{1}$ plan, (b) $(\mathrm{x}=-1,8 \mathrm{~cm}, \mathrm{z}=1,8 \mathrm{~cm}), \mathrm{xz}_{1}$ plan $(\mathrm{c})(\mathrm{y}=-2 \mathrm{~cm}, \mathrm{z}=8 \mathrm{~cm}), \mathrm{yz}_{3^{\prime}}$ plan

For example, 3 points are chosen on 2 different vertical plans. We notice that at the top of the cavity, where turbulence is developed, the velocity norm fluctuation seem random and to change rapidly in time and the values seem already at a steady state when data acquisition started, Fig. 11 (a). At the bottom of the enclosure, near the jet, fluctuation seem organized and almost periodic with a low frequency (period of a 100 seconds), Fig. 11 (b), this phenomenon is strange and the amplitude of the oscillation (around $5 \mathrm{~cm} / \mathrm{s}$ ) seem to indicate unsteady interaction with an unsteady ambient atmosphere in the garage entering the cavity. This might occur in the door is not properly closed, or acquisition starts soon after the smoke injection. At last we choose a point located at mid height near the jet, Fig. 11 (c), the time evolution seem to remain unsteady at 500s, 
with variations abnormal peaks of fluctuation around 220s and 450s. Such analysis of the time histogram indicates that this experiment is still unsteady after 500s. This conclusion is confirmed looking at the xz3' plan velocity norm 2D plot. All plots in constant $y$ plans should be symmetrical versus $x=0$ plan. We see that a structure clearly dominates on the left side while the should be symmetrical structure is smaller and slightly shifted to the right side.

As a conclusion, both time histograms of the velocity norm should be checked for steadiness and 2D plot in vertical plans parallel to the vents wall should be checked for symmetry. When unsteadiness is detected, a larger time sample is used until steadiness is reached. In a rare case where no steadiness is achieved, experiment is checked to understand the problem (mostly verticality of the set-up or accidental smoke generation during the experiment). Experiment is redone.

\section{B. Statistical errors}

for a local quantity varying with time $\tau(t)$, we define the time averaging operator $\langle\cdot\rangle_{t}$ between a starting time $t_{\text {start }}$ and en ending time $t_{\text {end }}$ :

$<\tau>_{t}=\frac{1}{t_{\text {end }}-t_{\text {start }}} \int_{t_{\text {start }}}^{t_{\text {end }}} \tau(t) d t$

The fluctuation $(\cdot)^{\prime}$ is given by:

$\tau^{\prime}(t)=\tau(t)-<\tau>_{t}$

The time autocorrelation $r\left(t^{\prime}\right)$ is:

$r\left(t^{\prime}\right)=\frac{<\tau^{\prime}(t) \cdot \tau^{\prime}\left(t+t^{\prime}\right)>_{t \prime}}{<\tau^{\prime 2}>_{t}}$

where $<\cdot>_{t \prime}=\frac{1}{t_{\text {end }}-t_{\text {start }}} \int_{t_{\text {start }}}^{t_{\text {end }}-t^{\prime}}(\cdot)(t) d t$.

The norm of the fluctuation is:

$\operatorname{rms}\left\{\tau^{\prime}\right\}_{t}=\left(\frac{1}{t_{\text {end }}-t_{\text {start }}} \int_{t_{\text {start }}}^{t_{\text {end }}}\left(\tau^{\prime}(t)\right)^{2} d t\right)^{1 / 2}$

And the statistical error for the time average is:

$\operatorname{Err}(\tau)=\frac{\operatorname{rms}\{\tau\}_{t}}{\sqrt{N}}$

where $N$ is the number of uncorrelated data used to calculate the average.

We will see next that we manage to obtain more than 400 uncorrelated velocity fields per experiment (a thousand actually for $1 \mathrm{~Hz}$ acquisition). The statistical error for the averages is then lower than 
$5 \%$ of the measured standard deviation of the quantity. The standard deviation of the velocity norm being lower than $50 \%$ in a large majority of the flow region, the statistical error never exceeds $2.5 \%$ of the average quantity. In fact it is the PIV measurement accuracy which is the limiting factor in term of global accuracy.

\section{Time correlations}

On a steady experiment, we calculate cross correlation functions for time evolutions of the vertical velocity at different heights of the vertical axis $x=y=0$. The same operation is also performed for the CFD calculations. For the experiments, we used the fast camera because PIV acquisitions at $1 \mathrm{~Hz}$ with the PIV camera show only random fluctuations the sample time being quite beyond the physical correlation time.

We plot vertical velocity time correlations, Fig. 12, at various heights of the vertical oz axis. We observe a fast decay of the correlation ranging from approximately $0.01 \mathrm{~s}$ up to $0.1 \mathrm{~s}$. This corresponds to the correlation time length of fast fluctuations (probably turbulence). Slow oscillations of periods around 0.5 up to $1 \mathrm{~s}$ are also observed. Even faster oscillations can be seen in the second curve, with periods around 0.02 up to $0.1 \mathrm{~s}$. A spectrum calculation of those correlations doesn't show pikes associated to an oscillatory behavior. Those fluctuations are mainly due to jet pseudo oscillatory behavior in the $z=4 \mathrm{~cm}$ region, where large eddies appear and the jet interacts with air entering the bottom vent. Larger times of acquisition would be necessary to see the correlations decrease to 0 , but it is impossible due to memory limitations of fast cameras. It is nevertheless confirmed by the absence of correlation on $1 \mathrm{~Hz}$ acquisition sequences. Our main goal was to ensure no pure oscillatory beavior would exist at the acquisition frequency of $1 \mathrm{~Hz}$.
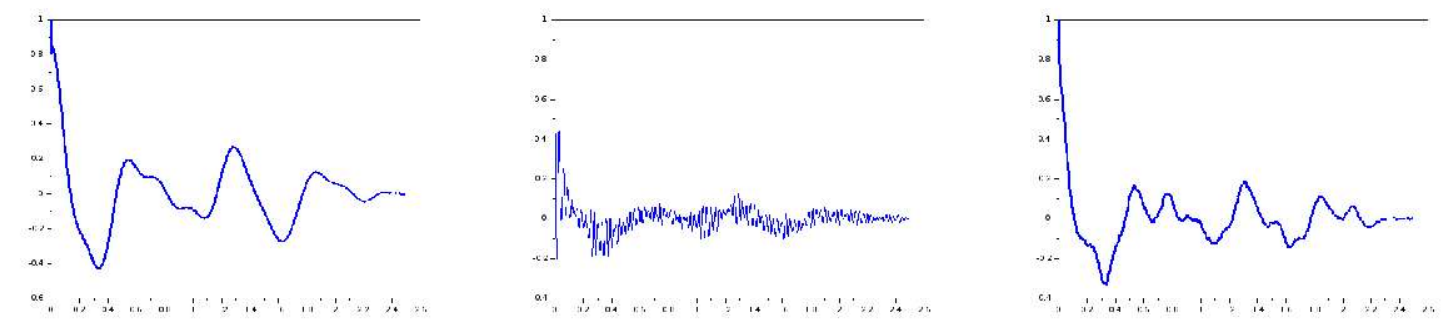

FIG. 12: Experiments time correlations of the vertical velocity at $x=y=0$ and respectively

$$
z=3 \mathrm{~cm}, z=6 \mathrm{~cm} \text { and } z=10 \mathrm{~cm}
$$

We conclude that correlation lengths for rapid fluctuation are below $0.1 \mathrm{~s}$ and intermediate correlation time exist around $0.5 \mathrm{~s}$ corresponding to larger interactions between the cavity and the outer domain. We therefore validate the fact that $1 \mathrm{~Hz}$ acquisition allows acquisition of statistically independent samples. The same work has been performed for CFD calculation, Fig. 13, and we draw similar conclusions with small scale correlation lengths being below $0.2 \mathrm{~s}$ and larger ones around 0.5 up to 1s. Although of interest, we won't analyse further the velocity correlations obtained with both approaches. It will be the subject of future works. 

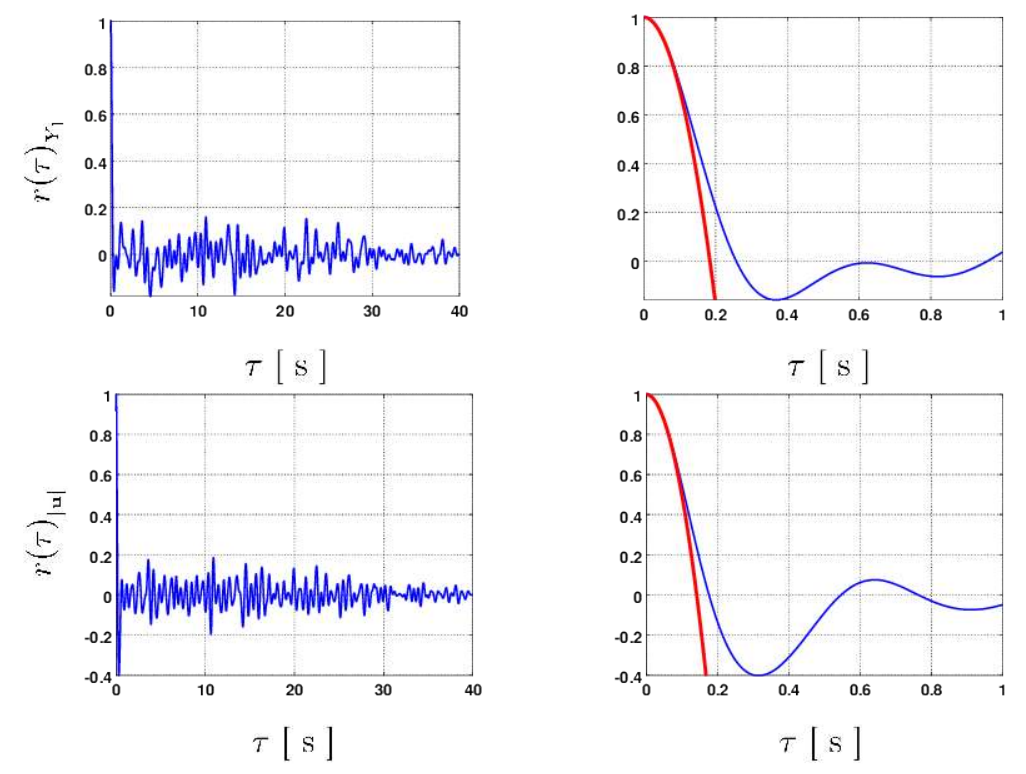

FIG. 13: Horizontal velocity time evolutions and time correlations at two different locations of the flow - CFD calculation

\section{COMPARISONS OF PIV RESULTS WITH L.E.S SIMULATIONS}

The objective of the section is to present comparisons with L.E.S simulations of this experiment provided by E. Saikali $[20,21]$. We first recall main characteristic of the CFD simulations as well as the quantities which are being compared. Then general characteristics of the flow are presented. At last quantitative comparisons between velocity profiles or $2 \mathrm{D}$ contour plots are presented.

\section{A. CFD modeling}

CFD calculations are well described in [20, 21]. We therefore just recall a few elements about the simulations. The CEA code TrioCFD [23] was used with the software plateform TRUST. The physical model is a low Mach approximation with Navier-Stokes equations for the flow and transport of the helium, perfect gas law, and both pressure and temperature are constant. Physical parameters dependence on the mixture composition is properly modeled. The L.E.S method relies on a Smagorinsky model for viscosity where the characteristic filter length is the cubic root of the cell volume.

The spatial discretization was a finite volume difference on a staggered grid with rectangular elements. A projection method is used for velocity and pressure decoupling. A two stages second order Rational Runge-Kutta scheme (RRK2) is employed for the temporal discretization. RRK2 scheme was first introduced by Wambecq in [24] to simulate steady flows and showed to be accurate, second order and A0 stable. Angrand et al. [25] show that RRK2 is more efficient than the first order Euler method, especially in cases where a central spacial scheme is considered. Convection terms are centered for the flow equations and uncentered (QUICK) for concentration equations, both are time explicit. Diffusive terms are implicit. Therefore time steps are imposed to respect 
the CFL criteria and range between $10^{-4} \mathrm{~s}$ and $3 \times 10^{-4} \mathrm{~S}$

The mesh, although composed of rectangular elements, is unstructured and realized with CEA cast $3 \mathrm{~m}$ code and converted with Salome open source plateform. Cells are about $0.7 \mathrm{~mm}$ wide which is about 3 times the Kolmogorov scale estimated by Saikali and about the same size as the PIV sub-windows. A thorough convergence study has been completed both for the discretization and also for the boundary conditions, applied to a selected meshed external domain. The study for the selection of a correct external domain is the main subject of the paper [20]. A view of the mesh is given, Fig. 14.
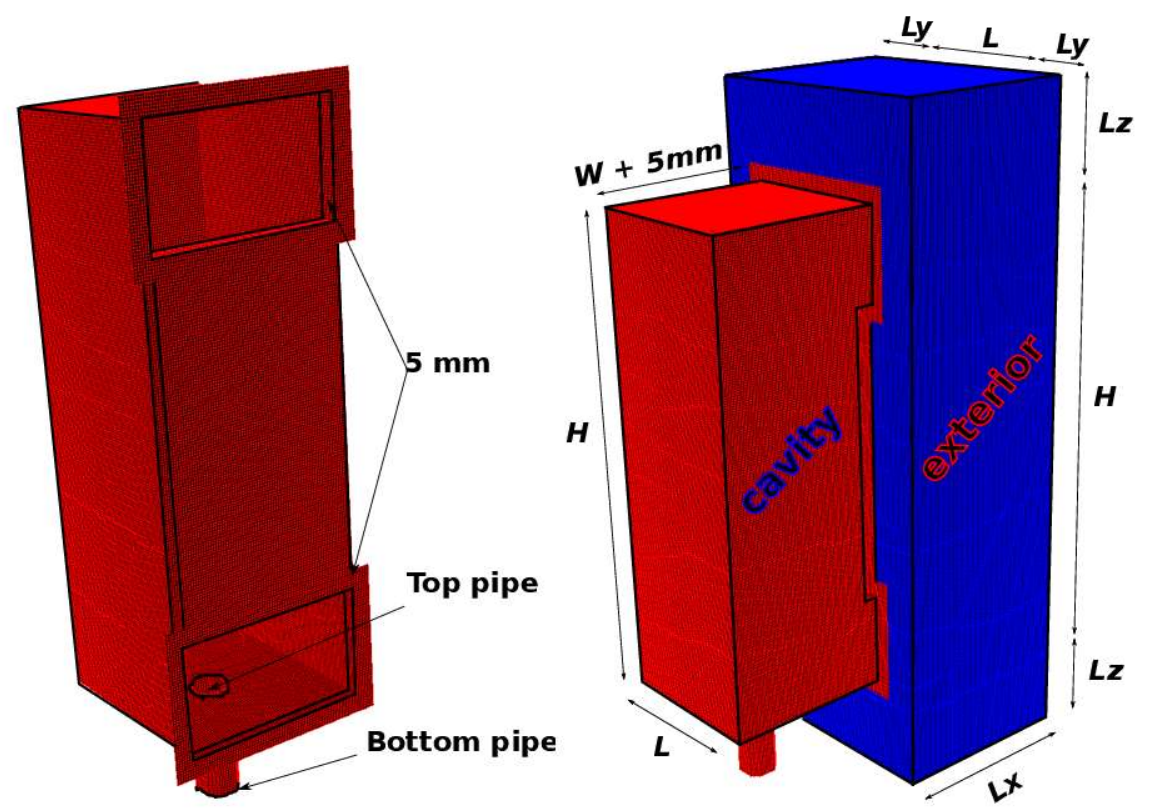

FIG. 14: Computational domain.

CEA TRUST-TrioCFD has been executed on a local cluster of CEA-SACLAY (Intel Xeon, E5$2680 \mathrm{~V} 2,2.8 \mathrm{GHz}, 128 \mathrm{Go}$ memory per node and infiniband QDR $40 \mathrm{Gbit} / \mathrm{s}$ ). MPI option has been specified for the parallel computation over 100 communicating processors, where the total mesh corresponds to $6.108484 \times 10^{6}$ cells. The simulation is carried on to 110 s of physical time. A steady state is proven to be reached after $40 \mathrm{~s}$, but data are only used between $80 \mathrm{~s}$ and $110 \mathrm{~s}$ to calculate the statistical quantities which is enough to have a negligible statistical error compared to the discretization error. L2 norm convergence study indicates that velocity should be converged at approximately $10 \%$ for $\mathrm{L}_{2}$ norm.

\section{B. Flow structure in the xoz plan}

Jet structure We are observing the flow structure in the xoz plan which is vertical, perpendicular to the vents and contains the $o z$ axis. We represent, Fig. 15, instantaneous pictures of the seeded flow as well as the time averaged velocity norm.

Black regions of the flow are corresponding to a lack of fog droplets and therefore higher helium 

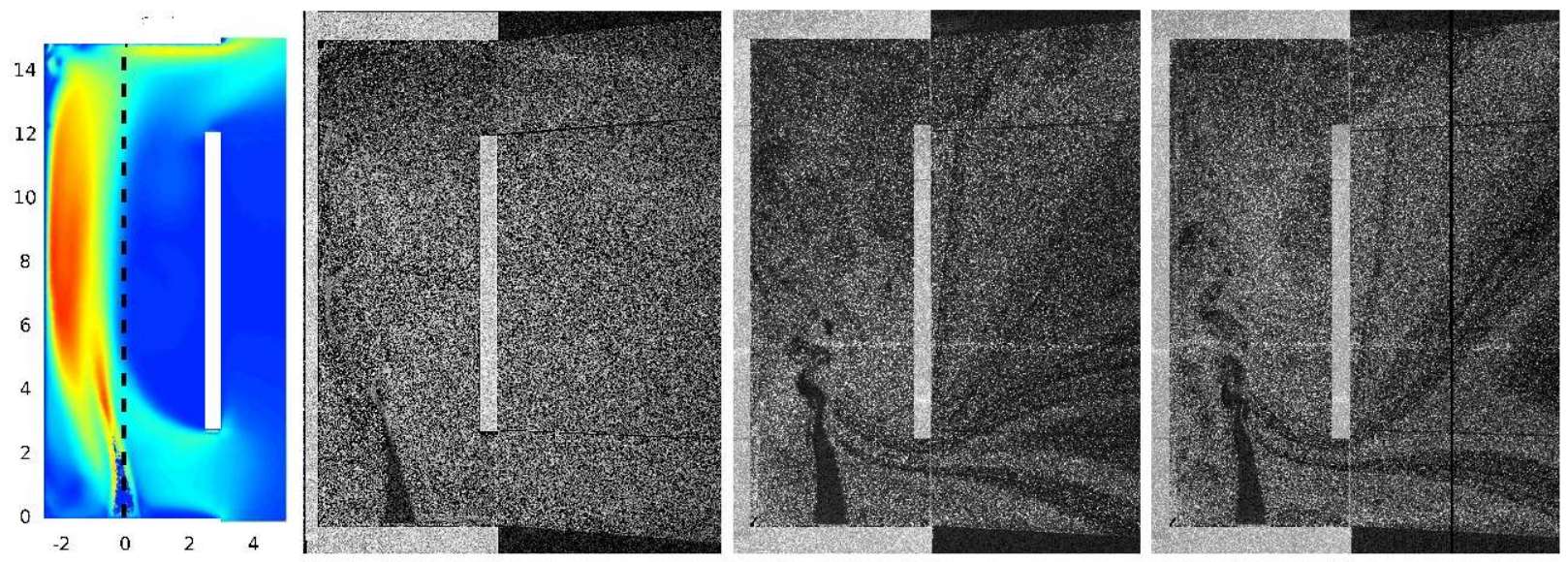

FIG. 15: Time averaged velocity norm on left picture (PIV), the 3 pictures on the right are instantaneous snapshot of the flow in the xoz plan at different times.

concentration (injected helium has no particles). Article resolution cannot allow to check at the seeding quality. We assert that $2 \mathrm{~cm}$ above the injection, seeding is enough to measure PIV velocity fields as show on the velocity plot.

We note that the level of fluctuations along the plume varies at different times. Nevertheless fluctuations start to develop slightly above the upper part of the bottom vent. We consider that a laminar to turbulent flow occur in the jet for a $z$ height ranging between 3 and $5 \mathrm{~cm}$. This instability seems to be the result of the interaction of air entering the bottom vent and bending the plume with unsteady structures development. Black structures are also seen along the left wall facing the vents, behind the plume. Those structures have to be understood as a "turning movement of the jet" due to the interaction of lateral entrance of air and vertical movement of the jet flow, illustrated in a top view from [20], Fig. 16.

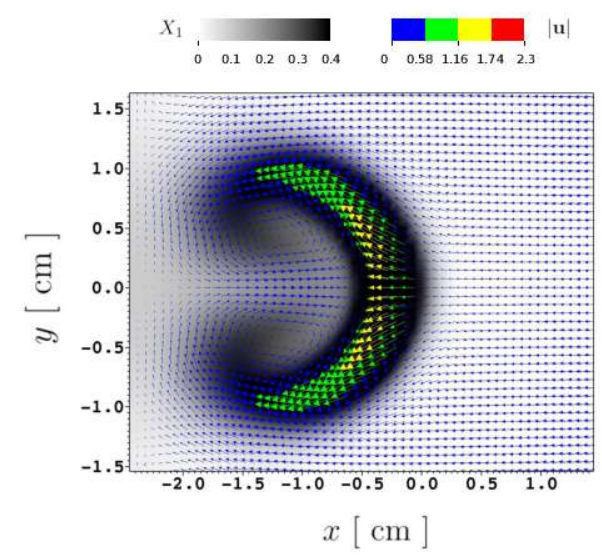

FIG. 16: Time averaged velocity norm field in a horizontal plan at $z=3 \mathrm{~cm}$. CFD calculation from Saikali [20]. This shows the interaction of the jet and the lateral flow entering the bottom vent.

It is important to note that a low Mach model is required on the CFD calculation, the interaction between entering air and ascending helium is strongly diminished if density ratios are not properly 
taken into account. Indeed, we also observe that the jet is inclined toward the back wall (left) facing the vents.

Comparison between PIV and CFD : We represent, Fig. 17, a comparison between PIV and CFD velocity norm in the $X o z$ plan.
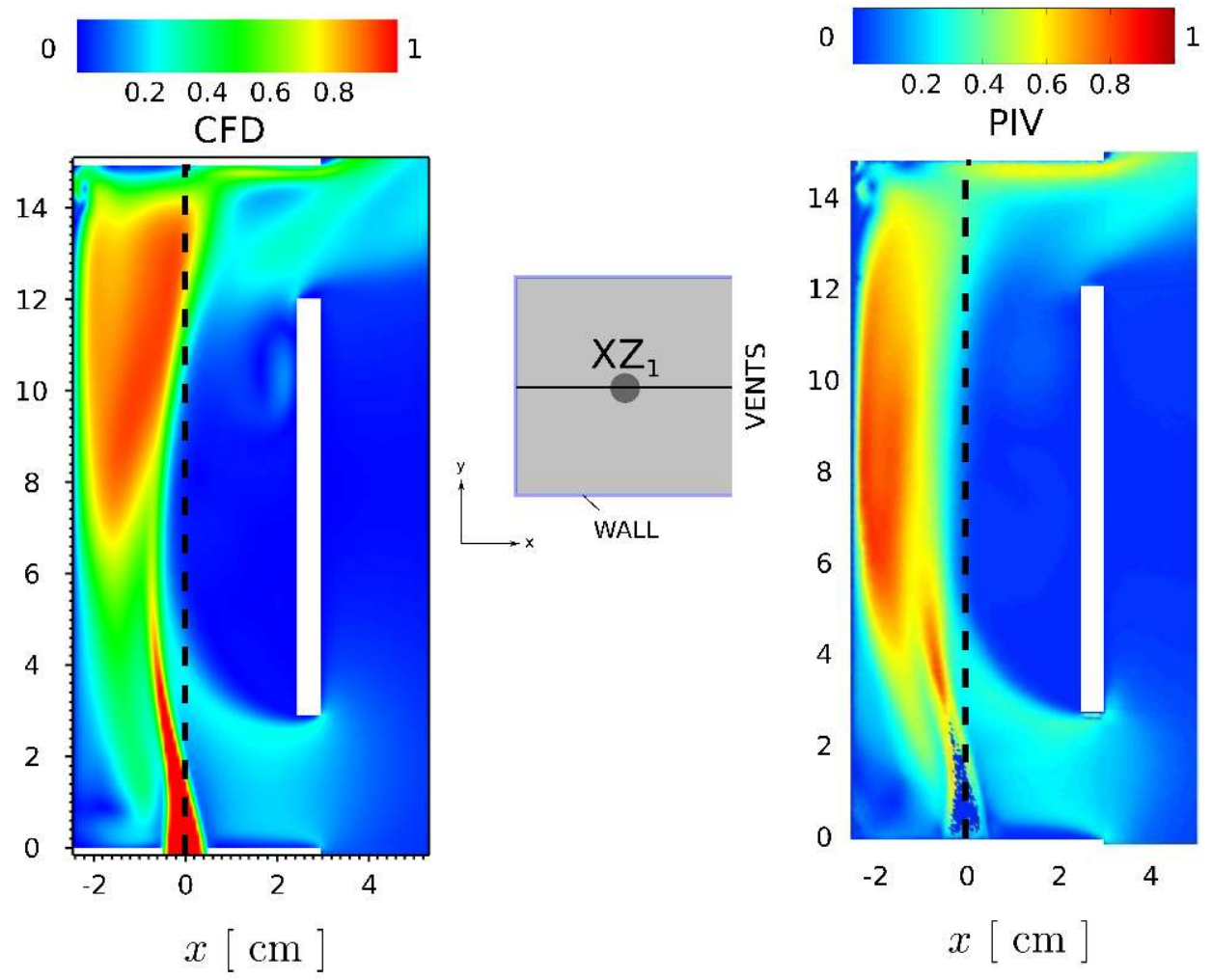

FIG. 17: Time averaged velocity field in the plan xoz. CFD calculations on the left and PIV results on the right.

To start with differences, we notice a stronger interaction of the jet and the left wall for the PIV measurements. That interaction starts earlier, approximately at $z=4 \mathrm{~cm}$ whereas this interaction develops at $z=7 \mathrm{~cm}$ for CFD. In order to have better understanding of those differences, we plot velocity norm profiles in the same plan at varying heights, Fig. 18. Velocity norm intensity differences might be as large as $20 \mathrm{~cm} / \mathrm{s}$ up to $z=7 \mathrm{~cm}$ and event larger at the top of the cavity. It is clearly above numerical and experimental expected errors. Nevertheless, the profiles show strong similarities: the main jet (from helium injection) sees its intensity decreasing while an ascending structure develops near the left wall. The main difference is that the main jet remains dominant in CFD while starting at $z=5.5 \mathrm{~cm}$ the wall structure becomes dominant in PIV. We will see later on that it is mainly due to a lack a fluctuation in the jet for CFD (or too much fluctuations for the experiment).

We also note strong similarities: the inclination of the jet is identical in both approaches. At the top wall, a similar thin jet layer is observed and exits the cavity through the top vent. A small eddy develops at the top left corner and another one develops near the bottom of the top vent, we illustrate this with a zoom on instantaneous vector fields Fig.19. 


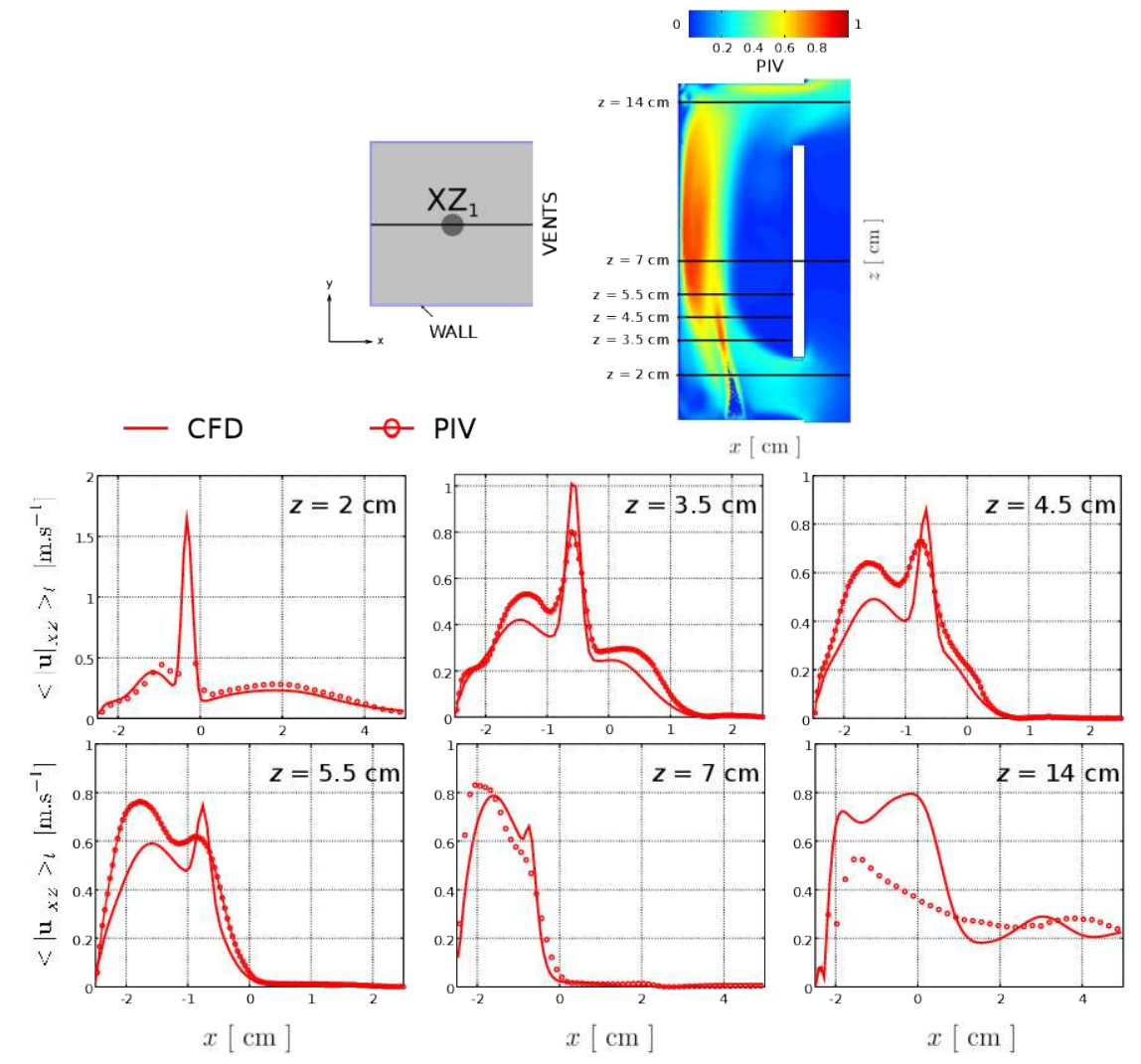

FIG. 18: Time averaged velocity norm profiles at different heights in the plan xoz: comparisons between CFD calculations and PIV results.

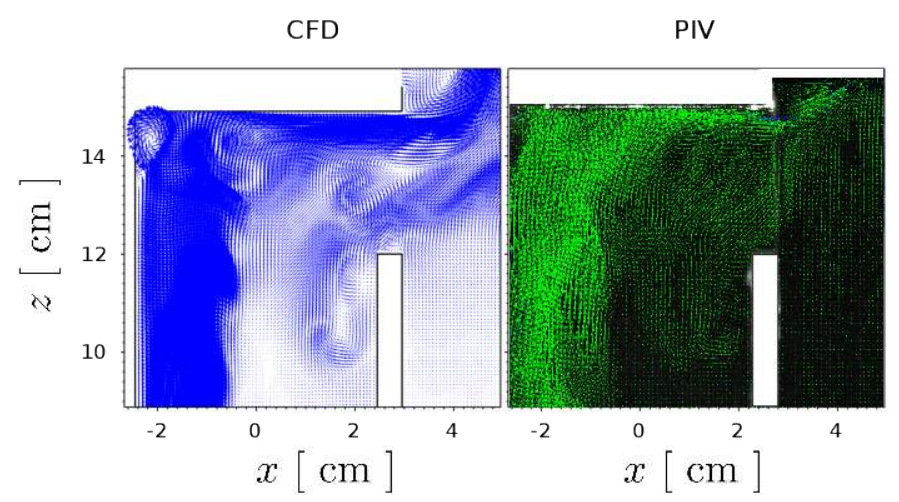

FIG. 19: Instantaneous velocity field in the plan xoz. CFD calculations on the left and PIV results on the right.

We also plot the horizontal time averaged velocity profiles at the bottom and the top vent, Fig. 20. Here again, strong similarities exist. We note at the bottom vent the same parabolic inverted profile. Velocities are stronger at the edge of the vent while smaller at the center. The absolute difference of velocity is around 3 to $4 \mathrm{~cm} / \mathrm{s}$. The higher measured PIV velocity is again due to higher turbulent rms fluctuations for the experimental results. The resulting higher mixing inside the cavity requires higher inflow of air. At the top vent, both profiles show the presence of an 
exiting jet layer, but its thickness is more important for the experiments.
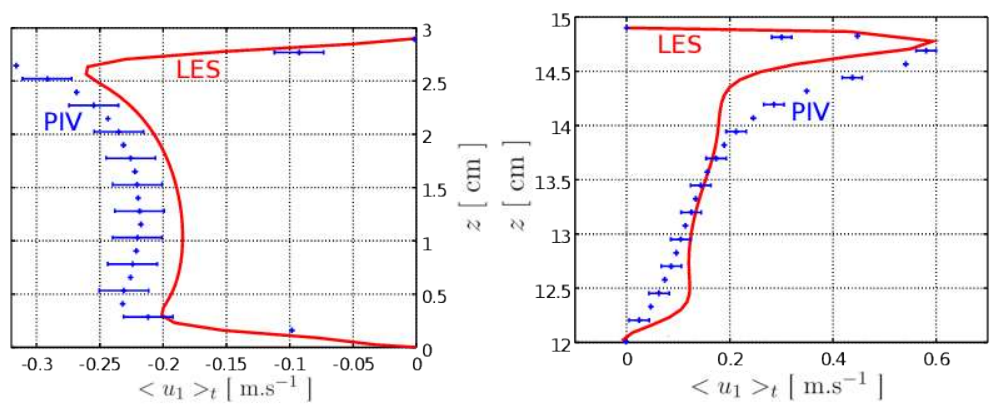

FIG. 20: Time averaged horizontal velocity profiles at the bottom and the top vent $x=5.5 \mathrm{~cm}$. PIV and CFD comparisons

\section{C. yz plans}

We now interest ourselves in plans parallel to the vent with a fixed $x$ position. We don't intend to reproduce all the results. In Fig. 21 we see that the contour structure of the velocity norm is qualitatively very similar for PIV and CFD.
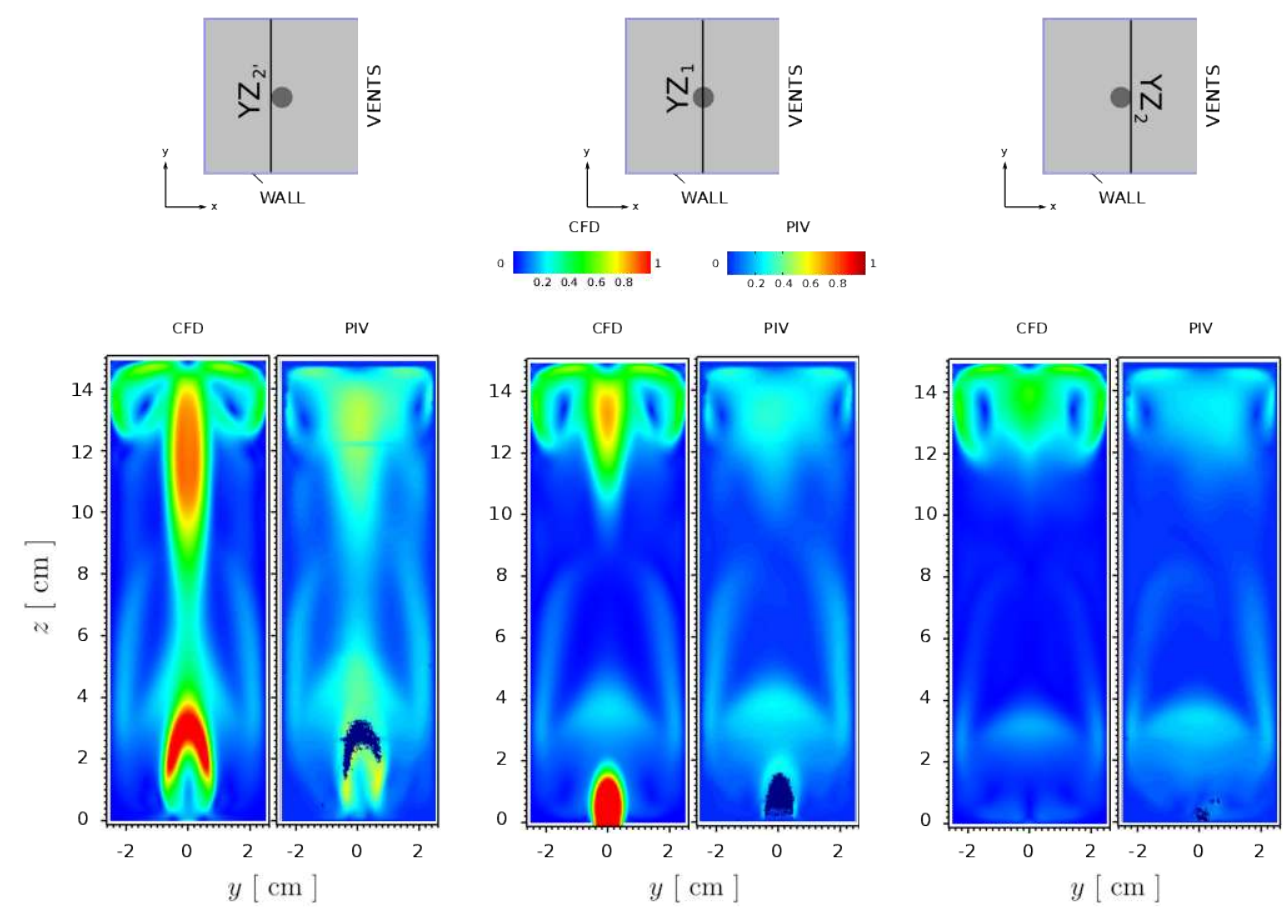

FIG. 21: Time averaged velocity norm contour plots in three yz vertical plans located at $x=-0.5 \mathrm{~cm}, x=0 \mathrm{~cm}, x=0.5 \mathrm{~cm}$. PIV and CFD comparisons

Differences are easily illustrated on velocity norm profiles, Fig. 22 where PIV and CFD profiles are quite similar at the bottom of the box but the main jet seems truncated (around $y=0$ and 
$z=13 \mathrm{~cm})$ for the PIV.

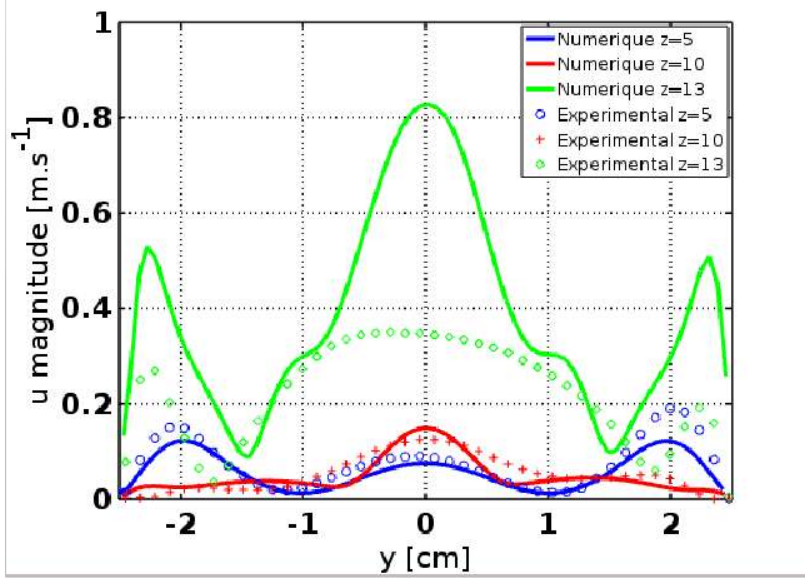

FIG. 22: Time averaged velocity norm profiles at different height of YZ1 vertical plan. PIV and CFD comparisons

We place the observation plan closer to vent, Fig. 23. We plot the corresponding velocity norm profiles at different heights, Fig. 24. Similar conclusions can be made. Nevertheless the profiles are a lot closer, since the main different between PIV and CFD are located in the region between the jet and the back wall facing the vent. The slightly higher velocity observed for PIV experiment at the lowest positions is due to a higher air velocity entering the bottom vent as shown in the previous section.
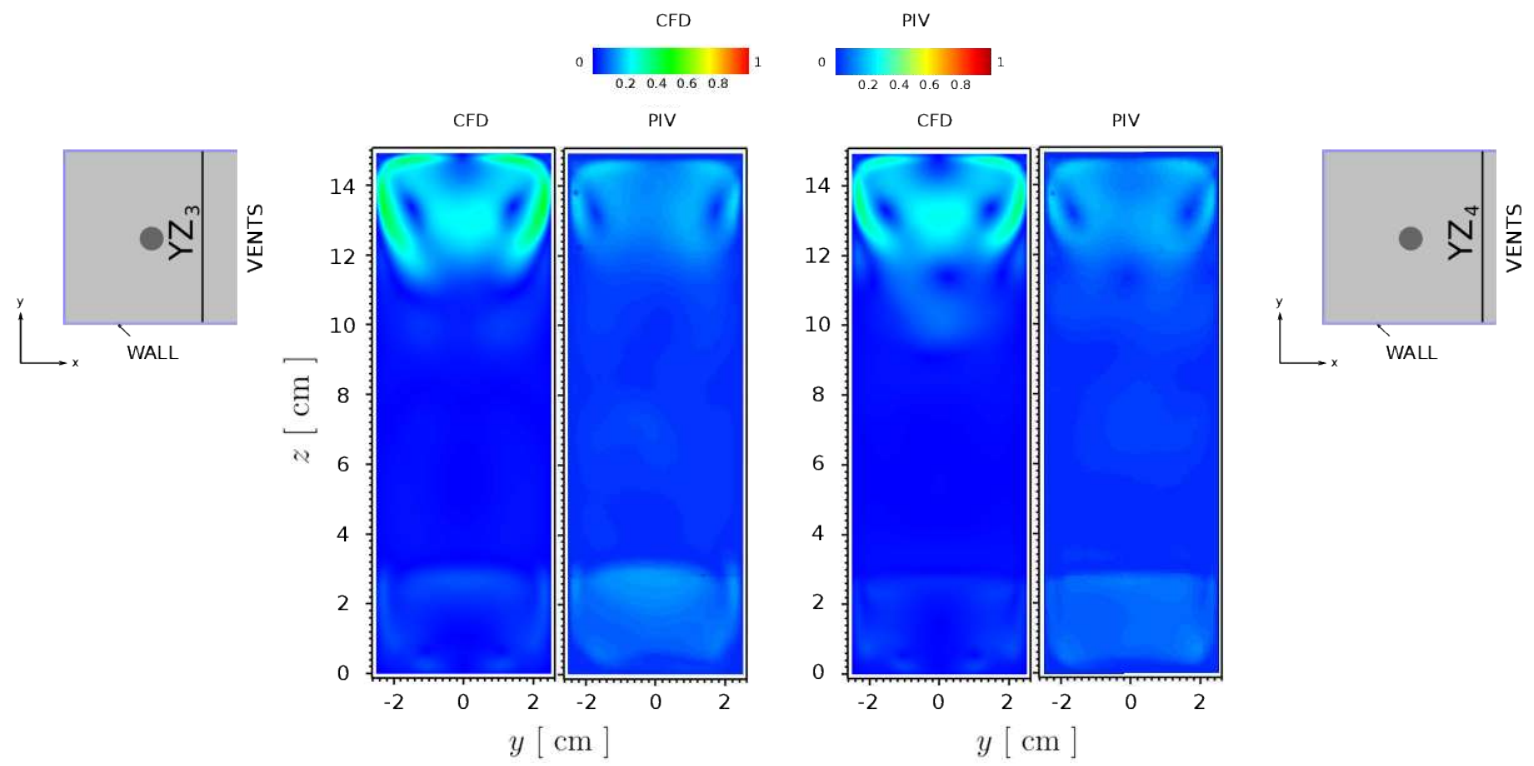

FIG. 23: Time averaged velocity norm contour plots in two yz vertical plans located at $x=1.5 \mathrm{~cm}$ and $x=2 \mathrm{~cm}$. PIV and CFD comparisons 


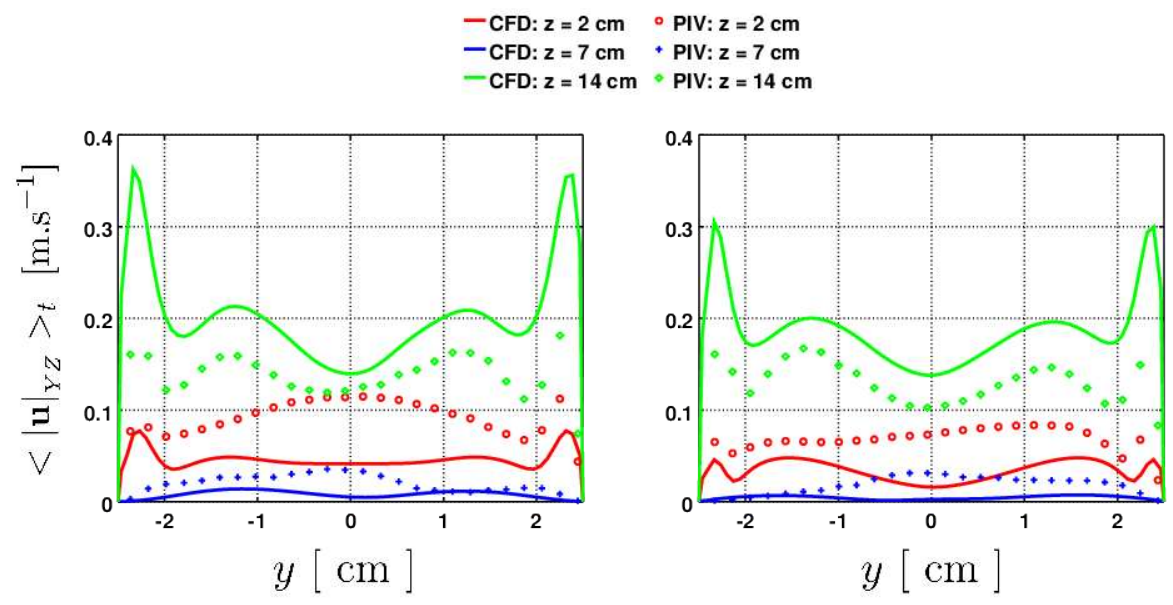

FIG. 24: Time averaged velocity norm contour profiles at different heights in two yz vertical plans located at $x=1.5 \mathrm{~cm}$ and $x=2 \mathrm{~cm}$. PIV and CFD comparisons

\section{RMS levels}

The main discrepencies in the results for PIV and CFD is actually due to a difference of fluctuation level in the jet for lower heights $z<7 \mathrm{~cm}$. This is illustrated by plotting the rms level of velocity fluctuations along the jet center, Fig. 25. Fluctuations are clearly at least 4 times higher in the experiments than in CFD calculations. Saikali personal communication indicates that a coarse DNS calculation clearly changes the level of fluctuations which becomes a lot closer than what observed on experiment.

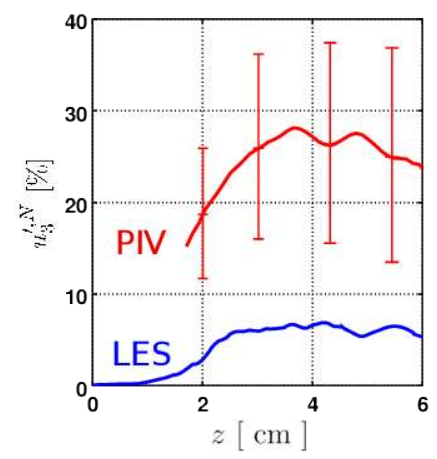

FIG. 25: Velocity fluctuations rms profile along the plume axis. PIV and CFD comparisons

On the other hand the general shape of the curve is similar, as shown Fig. 26, where rms fluctuations for LES are multiplied by 4 on the right figure. This indicates that laminar to turbulent transition occurs at the same height around $z=4 \mathrm{~cm}$ where fluctuations are at a maximum. As said, this will be the object of further work. 

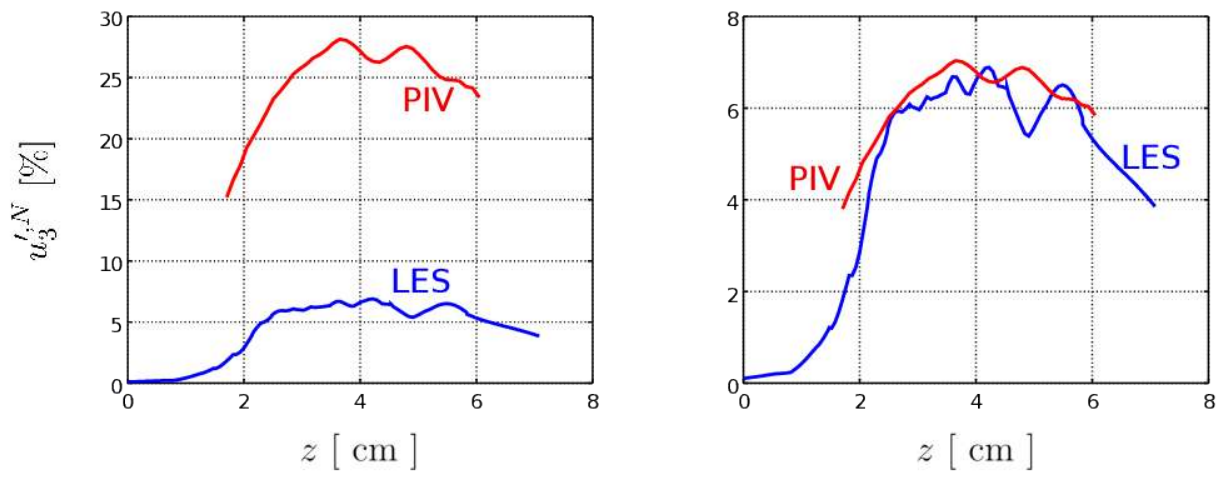

FIG. 26: Velocity fluctuations rms profile along the plume axis, scaled 4 times on the right for experiments. PIV and CFD comparisons

\section{E. Linden bi-layer structure}

As underlined in the introduction, it is difficult to explain the existence of Linden bi-layer structure without looking at the velocities. On the other side, it is difficult to assert that we are in presence or not of a bi-layer without looking at the concentrations. The reason why we want to assess a numerical method to model such experiments is that it is difficult to have access to both types of measurements simultaneously, whereas this is a standard result of the CFD simulations.

In the $Y Z 1$ plan, we draw the velocity field at the top of the cavity, Fig. 27. We observe on both results two eddies of approximately $3 \mathrm{~cm}$ width. This structure is typical of a Linden layer formation. However, Saikali [20] indicates that vertical concentration profiles contradicts the existence of such a layer due to the strong interaction between the jet and the left wall in xoz plan and recirculations near the top vent. The observed symmetrical eddies in the YZ1 plan are not strong enough to establish the bi-layer structure.
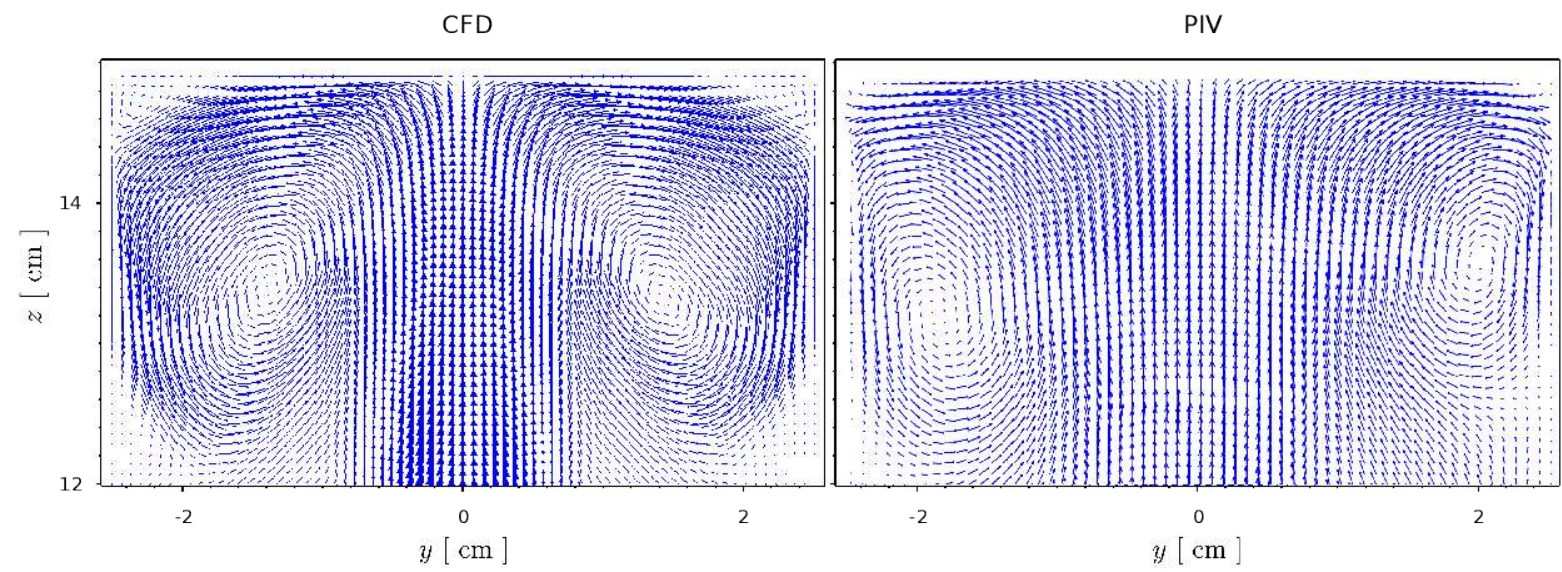

FIG. 27: Velocity vectors at the top of the cavity in YZ1 plan. PIV and CFD comparisons

We intentionally don't show the concentration profiles in [20] to underline the need for such a data in Linden structure analysis. 


\section{CONCLUSION}

Comparisons between the LES simulations and the PIV results are qualitatively in good agreement. Quantitative comparisons show more discrepancies, with similar contour shapes of the averaged quantities but significant differences in the spreading of the jet and its interaction with the back wall facing the vents. Overall, maximum velocities L2 errors are lower than $15 \%$ on average and L.E.S tend to over-predict the concentration due to reduced rms fluctuations and lower air flow entering through the bottom vent ( $15 \%$ lower).

It seems that finer grid (although we are at three times the Kolmogorov length) should be investigated as well as DNS calculations suggested by Saikali, even though the turbulent viscosity of Smagorinsky model is a third of the molecular viscosity.

On another side, experiments should be also performed on a modified geometry. Indeed we designed on purpose an experiment with a laminar to turbulent transition, vent and jet interaction and a jet to plume transition. This seems particularly unsteady, and we observed at many occasions that small disturbances in the experiments might strongly impact the results. Reproducibility was achieved at the price of a lot of effort. A less unstable type of experiment should be seeked for. This process in on going, with a slightly larger cavity and increased flow rate, which would produce a Linden bi-layer structure and hopefully more inertial turbulence which is easier to simulate.

At last, the understanding and the prediction of occurrence of a bi-layer structure in a two vented cavity needs to be improved. This requires improvement in the CFD modeling in order to produce reliable and accurate results: we are very close to it. On the experimental side, improvement should be done in instrumentation in order to have access simultaneously to velocity and concentration fields.

[1] G. Bernard-Michel and D. Houssin-Agbomson, Comparison of helium and hydrogen releases in $1 \mathrm{~m}$ 3 and $2 \mathrm{~m} 3$ two vents enclosures: Concentration measurements at different flow rates and for two diameters of injection nozzle, International Journal of Hydrogen Energy 42, 7542 (2017).

[2] G. Bernard-Michel, Rapport d'essais regroupant l'ensemble des résultats expérimentaux obtenus sur les rejets en milieu confiné (ec04), Rapport Technique (4.1).6.EC04, H2E (2012).

[3] G. Bernard-Michel, Rapport DM2S/SFME/LTMF RT/09-024, CEA (2009).

[4] E. Kaminski, G. Carazzo, and S. Tait, On the rise of turbulent plumes: quantitative effects of variable entrainment for submarine hydrothermal vents, terestrial and extra terrestrial explosive volcanism, Journal of geophysical research 113 (2008).

[5] B. Cariteau, Rapport DM2S/SFME/LEEF RT/2012-016/A, CEA (2012).

[6] G. R. Hunt and P. F. Linden, Displacement and mixing ventilation driven by opposing wind and buoyancy, J. Fluid Mech. 527, 27 (2005).

[7] B. Cariteau and I. Tkatschenko, Experimental study of the effects of vent geometry on the dispersion of a buoyant gas in a small enclosure, International Journal of Hydrogen Energy 38, 8030 (2013), ISSN 0360-3199.

[8] M. G. Worster and H. E. Huppert, Time-dependant density profiles in a filling, J. Fluid Mech. 132, 457 (1983).

[9] R. P. Cleaver, M. R. Marshall, and P. F. Linden, The build-up of concentration within a single enclosed volume following a release of natural gas, J. Hazardous Mater. 36, 209 (1984). 
[10] P. F. Linden, G. F. Lane-Serff, and D. A. Smeed, Emptying filling boxes : the fluid mechanics of natural ventilation, J. Fluid Mech. 212, 309 (1990).

[11] A. Venetsanos, E. Papanikolaou, B. Cariteau, P. Adams, and A. Bengaouer, Hydrogen permeation from $\{C G H 2\}$ vehicles in garages: $\{C F D\}$ dispersion calculations and experimental validation, International Journal of Hydrogen Energy 35, 3848 (2010), ISSN 0360-3199.

[12] G. Bernard-Michel, J. Trochon, E. Vyazmina, and O. Gentilhomme, Tech. Rep., Technical Report DEN/DANS/ DM2S/STMF/LIEFT/RT/12/020/A., CEA Saclay DEN/DANS/DM2S/STMF /LIEFT (2012).

[13] G. Bernard-Michel, B. Cariteau, J. Ni, S. Jallais, E. Vyazmina, D. Melideo, D. Baraldi, and A. Venetsanos, in Proceedings of ICHS 2013 (2013).

[14] G. Bernard-Michel, V. Molkov, E. Vyazmina, P. Hooker, D. Baraldi, and Venetsanos (CEA technical report, 2015).

[15] S. Giannissi, V. Shentsov, D. Melideo, B. Cariteau, D. Baraldi, A. Venetsanos, and V. Molkov, Cfd benchmark on hydrogen release and dispersion in confined, naturally ventilated space with one vent, International Journal of Hydrogen Energy 40, 2415 (2015), ISSN 0360-3199, URL http: //www.sciencedirect.com/science/article/pii/S0360319914033485.

[16] V. Molkov and V. Shentsov, Numerical and physical requirements to simulation of gas release and dispersion in an enclosure with one vent, International Journal of Hydrogen Energy 39, 13328 (2014), ISSN 0360-3199, URL http://www.sciencedirect.com/science/article/pii/S0360319914018977.

[17] S. Giannissi, J. Hoyes, B. Chernyavskiy, P. Hooker, J. Hall, A. Venetsanos, and V. Molkov, Cfd benchmark on hydrogen release and dispersion in a ventilated enclosure: Passive ventilation and the role of an external wind, International Journal of Hydrogen Energy 40, 6465 (2015), ISSN 0360-3199.

[18] HyIndoor, Pre normative research on the indoor use of fuel cells and hydrogen systems project website, http://www.hyindoor.eu/.

[19] E. Saikali, A. Sergent, G. Bernard-Michel, and C. Tenaud, in Proceeding of the 23ème Congrès Français de Mécanique, Lille, France (2017).

[20] E. Saikali, G. Bernard-Michel, A. Sergent, C. Tenaud, and R. Salem, Highly resolved large eddy simulations of an air-helium buoyant jet in a two-vented enclosure: influence of the computational domain, Int. Journal H2 Energy. Special edition for ICHS 2017. To be published (2018).

[21] E. Saikali, IFD, ED391, UPMC Paris 06, F-75006, Paris, France., PhD. Sorbonne Universités (2018).

[22] C. J. Chen and W. Rodi, Vertical turbulent buoyant jets: a review of experimental data, Nasa STI/Recon Technical Report A 80 (1980).

[23] P. Angeli, U. Bieder, and G. Fauchet, in Proceedings of 16th International Topical Meeting on Nuclear Reactor Thermal Hydraulics (NURETH-16), Chicago, USA (2015).

[24] A. Wambecq, Rational runge-kutta methods for solving systems of ordinary differential equations, Computing 20, 333 (1978).

[25] F. Angrand, V. Billey, A. Dervieux, J. Desideri, J. Periaux, and B. Stoufflet, On the use of rational Runge-Kutta methods in Euler steady-state computations (Springer Berlin Heidelberg, Berlin, Heidelberg, 1985), pp. 77-81, ISBN 978-3-540-39144-9, URL http://dx.doi .org/10.1007/3-540-13917-6_ 112. 